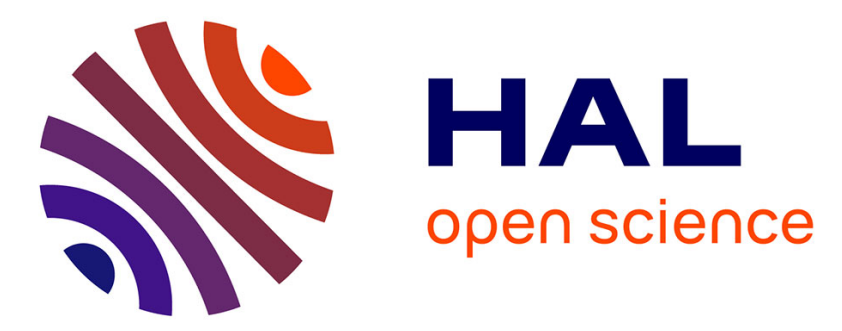

\title{
Inverse Design Approach for Low-Boom Supersonic Configurations
}

\author{
Andrea Minelli, Itham Salah El Din, Gérald Carrier
}

\section{To cite this version:}

Andrea Minelli, Itham Salah El Din, Gérald Carrier. Inverse Design Approach for Low-Boom Supersonic Configurations. AIAA Journal, 2014, 52 (10), pp.2198 - 2212. 10.2514/1.J052834 . hal01082591

\section{HAL Id: hal-01082591 \\ https://hal.science/hal-01082591}

Submitted on 13 Nov 2014

HAL is a multi-disciplinary open access archive for the deposit and dissemination of scientific research documents, whether they are published or not. The documents may come from teaching and research institutions in France or abroad, or from public or private research centers.
L'archive ouverte pluridisciplinaire HAL, est destinée au dépôt et à la diffusion de documents scientifiques de niveau recherche, publiés ou non, émanant des établissements d'enseignement et de recherche français ou étrangers, des laboratoires publics ou privés. 


\title{
Inverse Design Approach for Low-Boom Supersonic Configurations
}

\author{
Andrea Minelli*, Itham Salah el $\operatorname{Din}^{\dagger}$ and Gérald Carrier ${ }^{*}$
}

The shaped sonic boom theory is a valuable, efficient, computationally economical and robust tool in preliminary design of low-boom aircraft configurations. Instead of introducing a new F-function parameterization, as it has been investigated already in the past, the paper adopts a more general formulation proposed in the literature and focuses on reducing the limitations of the inverse method in the design process. Three main contributions are proposed: 1) a revisited procedure based on optimization to solve the coefficients of the F-function that enables to switch between different parameterizations; 2) a definition of the geometry corresponding to the equivalent area distribution combined with a fuselage tailoring process based on direct shape optimization; 3) a strategy to introduce a generic acoustic metrics in the definition of an optimum F-function. The proposed strategy enables the designer to evaluate the geometry of a low-boom configuration that corresponds to a desired F-function in a complete inverse design approach. In this way, the usual limits of the inverse method are significantly alleviated by the present method.

\footnotetext{
*PhD student, ONERA, The French Aerospace Lab, Andrea.Minelli@ onera.fr

${ }^{\dagger}$ Research Engineer, ONERA, The French Aerospace Lab, Itham.Salah_El_Din@onera.fr

¥Research Engineer, ONERA, The French Aerospace Lab, Gerald.Carrier@ onera.fr
} 


\section{Nomenclature}

\begin{tabular}{|c|c|c|}
\hline$a$ & $=$ & local speed of sound, $\mathrm{m} / \mathrm{s}$ \\
\hline$a_{h}$ & $=$ & local speed of sound at altitude $h, m / s$ \\
\hline$A$ & $=$ & Area of ray tube, $m^{2}$ \\
\hline$A_{e}$ & $=$ & Equivalent area, $m^{2}$ \\
\hline$A_{g}$ & $=$ & Geometrical area distribution, $m^{2}$ \\
\hline$A_{h}$ & $=$ & Area of ray tube at altitude $h, m^{2}$ \\
\hline$A_{l}$ & $=$ & Lift term of the equivalent area, $m^{2}$ \\
\hline$A_{v}$ & $=$ & Volume term of the equivalent area, $m^{2}$ \\
\hline AIDA & $=$ & Acoustic Inverse Design Approach \\
\hline$B, \beta_{0}, \beta_{1}$ & $=$ & F-function coefficients, $m^{-0.5}$ \\
\hline$C, D, H$ & $=$ & F-function coefficients, $m^{0.5}$ \\
\hline$c_{p}$ & $=$ & Pressure coefficient, $c_{p}=\frac{p-p_{\infty}}{\frac{1}{2} \rho_{\infty} U_{\infty}^{2}}$ \\
\hline CFD & $=$ & Computational Fluid Dynamics \\
\hline$F$ & $=$ & Whitham F-function, $m^{0.5}$ \\
\hline$h$ & $=$ & Cruise altitude, $m$ \\
\hline$J_{A_{e}}$ & $=$ & Objective function based on equivalent area \\
\hline$J_{F}$ & $=$ & Objective function based on F-function \\
\hline$J_{P}$ & $=$ & Objective function based on ground signature \\
\hline$J_{\text {comb }}$ & $=$ & Combined objective cost function \\
\hline$l$ & $=$ & Lift per unit length, $N / m$ \\
\hline$L$ & $=$ & Aircraft body length, $m$ \\
\hline$M_{\infty}$ & $=$ & Mach number \\
\hline$M_{h}$ & $=$ & Mach number at altitude $h$ \\
\hline$N, P$ & $=$ & Number of segments in the F-function before and after $x=\lambda$ \\
\hline$n$ & $=$ & Number of optimization variables \\
\hline$p$ & $=$ & Local pressure, $P a$ \\
\hline$p_{h}$ & $=$ & Local pressure at altitude $h, P a$ \\
\hline$p_{\infty}$ & $=$ & Free stream pressure, $P a$ \\
\hline
\end{tabular}




$$
\begin{array}{ll}
P_{i} & =\text { i-th component of the projected gradient } \\
P_{f} & =\text { Bow shock amplitude, } P a \\
P L d B & =\text { Perceived loudness deciBel } \\
P_{r} & =\text { Rear shock amplitude, } P a \\
r & =\text { Radial co-ordinate, } m \\
s & =\text { Slope of the balancing area line, } m^{-0.5} \\
t & =\text { F-function coefficient, } m \\
U_{\infty} & =\text { Free stream velocity, } m / s \\
x, \tau & =\text { Axial co-ordinate in the body axis, } m \\
x_{e} & =\text { Horizontal axis co-ordinate, } m \\
y_{f} & =\text { Nose bluntness parameter, } m \\
y_{r} & =\text { Intersection of the F-function and the rear balancing line, } m \\
W & =\text { Aircraft weight, } \mathrm{kg} \\
\beta & =\text { Prandtl-Glauert factor } \sqrt{M_{\infty}^{2}-1} \\
\eta, \xi & =\text { F-function variables } \\
\gamma & =\text { Specific heat capacity } \\
\mu & =\text { Mach angle, degree } \\
\rho_{\infty} & =\text { Free stream density, } \mathrm{kg} / \mathrm{m}^{3} \\
\theta & =\text { Azimuth angle, degree } \\
\lambda & =\text { Wing trailing edge location, } m \\
\Lambda & =\text { Age parameter, } m^{0.5} \\
&
\end{array}
$$

\section{Introduction}

Minimizing the sonic boom has been the focus of an important investigation effort since the 1950's. Multiple approaches have been analyzed and developed in order to reduce this deleterious phenomenon. Despite countless studies ( [1]- [8]), a low boom shape allowed to fly overland without restrictions has not been identified yet. Detailed reviews of the state of the art in sonic boom modeling and minimization techniques are provided by Plotkin [9] and Alonso [10] . Most studies are based on the Whitham corrected supersonic linearised theory [11] and its extension to lifting 
bodies proposed by Walkden [12]. The acoustic disturbances generated by a supersonic aircraft are modeled considering the distribution of sources and sinks along an equivalent body of revolution expressed through an F-function formulated as follows:

$$
F(\tau, \theta)=\frac{1}{2 \pi} \int_{0}^{\tau} \frac{A_{v}^{\prime \prime}(x)}{\sqrt{\tau-x}} d x+\frac{\beta \cos \theta}{\rho_{\infty} U_{\infty}^{2}} \frac{1}{2 \pi} \int_{0}^{\tau} \frac{l^{\prime}(x)}{\sqrt{\tau-x}} d x
$$

where $A_{v}$ is the cross section area distribution obtained by intersection of the geometry with a plane inclined at the Mach angle $\mu$ defined as $\operatorname{asin}(1 / M)$ with respect to the flight trajectory after projection normally to the flight axis (see Fig. 1), and $l^{\prime}$ defines the lift per unit length derivative with respect to the flight axis coordinate.

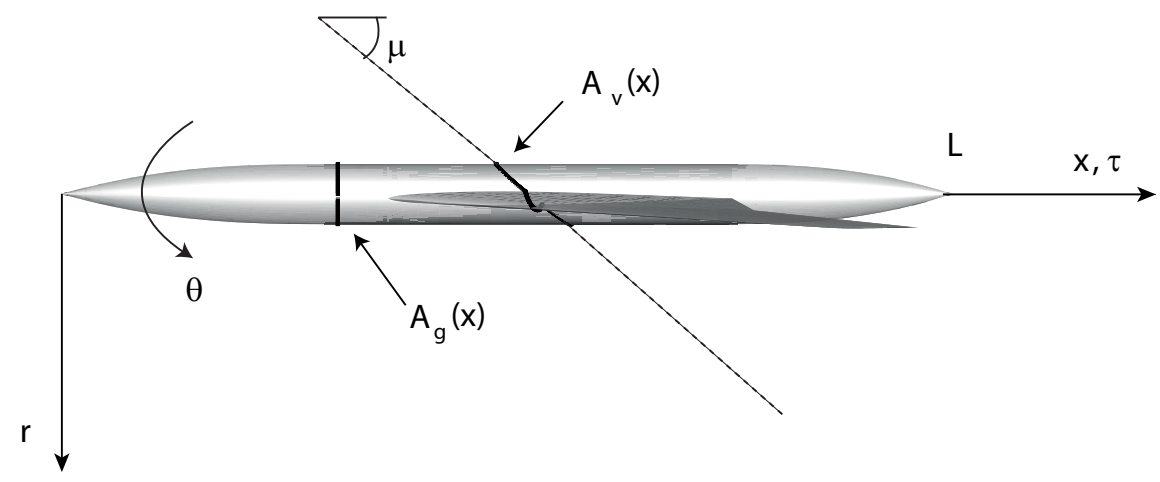

Figure 1. Nomenclature definitions.

Let $A_{l}$ be defined as:

$$
A_{l}(\tau, \theta)=\frac{\beta \cos \theta}{\rho_{\infty} U_{\infty}^{2}} \int_{0}^{\tau} l(x) d x
$$

such that an equivalent area distribution can be expressed as the sum of the volume and the lift terms $A_{e}=A_{v}+A_{l}$, allowing a compact formulation of the F-function:

$$
F(\tau, \theta)=\frac{1}{2 \pi} \int_{0}^{\tau} \frac{A_{e}^{\prime \prime}(x, \theta)}{\sqrt{\tau-x}} d x
$$

Within the hyphothesis that the flow field is linear between the aircraft and the location at which the pressure signature is computed, the pressure around the aircraft is explicitly related 
to the F-function, the distance from the source $r$, and an azimuth $\theta$ :

$$
\Delta P(\tau, \theta, r)=p_{\infty} \frac{\gamma M_{\infty}^{2}}{\sqrt{2 \beta r}} F(\tau, \theta)
$$

The Whitham theory provides a good approximation of the sonic boom overpressure as long as the Mach number remains below $M_{\infty}=3$ ( [13], [14]). In the case of highly non-linear and three dimensional flow behavior, typically close to the aircraft and in accelerated flight, a nonlinear CFD based method should be adopted. Indeed, CFD provides the pressure perturbations allowing the F-function calculation from (Eq. (4)). Cheung et al. [15] proposed in the 1990's the use of a parabolised Navier-Stokes equations solver to predict the flow field farther than what is typically affordable with classical RANS solvers. Numerical computations of the far field using Euler and full-potential equations combined with mesh adaptation have been evaluated on simple configurations by Kandil et al. [16]. Mesh adaptation has become a necessity to obtain accurate pressure signatures far from the source ( [17], [18], [19]). In Loseille and Lohner [20] the first direct sonic boom evaluation with a single-layer approach is performed using such advanced mesh adaptation techniques. The pressure signature is evaluated at various distances from the aircraft to the ground. Another approach formulated by Plotkin and Page [21] consists in an asymptotic multipole decomposition of the pressure disturbances source to comply with the local axisymmetry assumption needed for classical acoustic propagation.

Direct shape optimization is today widely used to design supersonic configurations ( [22], [23], [24], [25]). The objective of the present research is to introduce low-boom criteria into aircraft conceptual design based on an optimization framework. In preliminary design phases, such approaches may require many configuration analyses and can be prohibitively expensive, if based on high fidelity methods. An alternative to the direct approach is to reach a target ground signature using an inverse design process, which is the focus of the present paper. This approach is based on the shaped sonic boom theory [26] and Whitham's theory, providing a suitable equivalent area distribution. The lack of relationship between the equivalent area distribution and the real geometry of the configuration is one of the main drawbacks of the approach. Approximate analytical methods ( [27], [28]) have shown convergence problems of the algorithm in case of 
geometrical discontinuities. A first step toward a complete inverse approach is the use of shape modification technique to obtain a target equivalent area ( [29], [30]) which may be reached by an adjoint approach as in Palacios [31].

In the present paper, the latest developments of the shaped sonic boom theory are briefly reviewed as an introduction to the presentation of the inverse design tool AIDA developed at ONERA as well as its validation process. The definition of the geometrical area distribution that corresponds to the equivalent area distribution is investigated and a fuselage tailoring process is proposed. An original procedure taking into account an acoustic metric in the inverse design process is described. Finally, the application of the present inverse design optimization approach to the fuselage shape tailoring of a wing-body configuration is then analyzed. The impact on the solution of the choice of parameterization as well as target objective function is analyzed.

\section{The shaped sonic boom theory}

The F-function represents the disturbances near the source and is explicitly related to the geometry and the acoustic signal. The objective of the shaped sonic boom theory is to tailor the F-function to generate a target shaped ground signature. This numerical approach has been recently validated with in-flight measurements ( [32], [33]).

The results of the studies conducted by Petty [34], Hayes [35], Howell [36], George [37] and Jones [38] converge toward an F-function parameterization defined by George and Seebass [26]. This parameterized F-function can then be designed to produce a ground signature with specific characteristics such as minimum shock overpressure, impulse or shock pressure rise. Darden [39] proposed a modification of the model to consider nose bluntness relaxation to make it compliant with acceptable drag. Despite the introduction of new parameters the Darden F-function parameterization is not able to describe all possible generic configurations. The High Speed Research (HSR) program ( [4], [5]) studies show that more complex F-function modelling is needed to achieve low-boom signatures . A first generalization was proposed by Rallabhandi and Mavris [40] aiming 


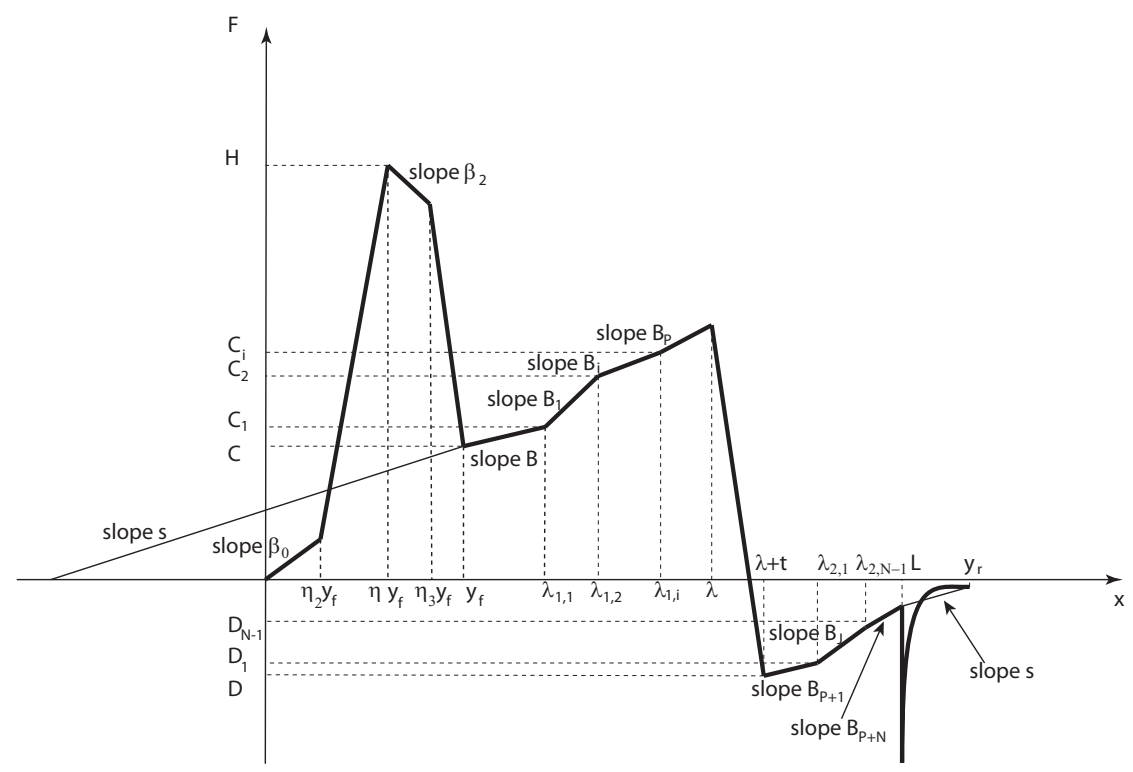

Figure 2. Plotkin et al. [43] F-function parameterization.

at considering low-boom signatures described in the work of Mack [41]. Further improvements of the parameterization were proposed by Haas and Kroo [42] and Plotkin et al. [43]. Haas and Kroo revisited the classical shaped sonic boom theory to consider multi-shock signatures and primordial elements of trade-off between aerodynamics and acoustics.

The two methods are very similar, both aim at generalizing the sonic boom minimization theory considering more general ground signature thus increasing the design space in terms of potential configuration designs. Due to its similarity with the classical formulation, the F-function parameterization described by Plotkin is analyzed (see Fig. 2) and adopted in this work. The F-function 
can be expressed as:

$$
F(x)=\left\{\begin{array}{lc}
\beta_{0} x & 0 \leq x \leq \eta_{2} y_{f} \\
\frac{H-\beta_{0} \eta_{2} y_{f}}{\left(\eta-\eta_{2}\right) y_{f}} x-\frac{H-\beta_{0} \eta y_{f}}{\left(\eta-\eta_{2}\right) y_{f}} \eta_{2} y_{f} & \eta_{2} y_{f} \leq x \leq \eta y_{f} \\
\beta_{2}\left(x-\eta y_{f}\right)+H & \eta y_{f} \leq x \leq \eta_{3} y_{f} \\
\frac{C-H-\beta_{2}\left(\eta_{3}-\eta\right) y_{f}}{\left(1-\eta_{3}\right) y_{f}}\left(x-\eta_{3} y_{f}\right)+H+\beta_{2}\left(\eta_{3}-\eta\right) y_{f} & \eta_{3} y_{f} \leq x \leq y_{f} \\
B_{1}\left(x-y_{f}\right)+C & y_{f} \leq x \leq \lambda_{1,1} \\
B_{2}\left(x-\lambda_{1,1}\right)+C_{1} & \lambda_{1,1} \leq x \leq \lambda_{1,2} \\
\cdots & \\
B_{P}\left(x-\lambda_{1, P-1}\right)+C_{P-1} & \lambda_{1, P-1} \leq x \leq \lambda \\
-\frac{D+C_{P}}{t}(x-\lambda)+C_{P} & \lambda \leq x \leq \lambda+t \\
B_{P+1}(x-\lambda-t)-D & \lambda+t \leq x \leq \lambda_{2,1} \\
B_{P+2}\left(x-\lambda_{2,1}\right)-D_{1} & \lambda_{2,1} \leq x \leq \lambda_{2,2} \\
\cdots & \\
B_{P+N}\left(x-\lambda_{2, N-1}\right)-D_{N-1} & \lambda_{2, N-1} \leq x \leq L
\end{array}\right.
$$

The equivalent area distribution can be found using an Abel transformation:

$$
A_{e}(L, 0)=\frac{\beta W}{\rho U_{\infty}^{2}}=4 \int_{0}^{L} F(\tau, 0) \sqrt{L-\tau} d \tau
$$


The equivalent area distribution is written as:

$$
\begin{aligned}
A_{e}(x, 0)= & \frac{16}{15} \beta_{0} x^{5 / 2}+1\left(x-\eta_{2} y_{f}\right) \frac{16}{15}\left(x-\eta_{2} y_{f}\right)^{5 / 2}\left[\frac{H-\beta_{0} \eta_{2} y_{f}}{\left(\eta-\eta_{2}\right) y_{f}}-\beta_{0}\right]+1\left(x-\eta y_{f}\right) \frac{16}{15}\left(x-\eta y_{f}\right)^{5 / 2}\left[\beta_{2}-\right. \\
& \left.\frac{H-\beta_{0} \eta_{2} y_{f}}{\left(\eta-\eta_{2}\right) y_{f}}\right]+1\left(x-\eta_{3} y_{f}\right) \frac{16}{15}\left(x-\eta_{3} y_{f}\right)^{5 / 2}\left[\frac{C-H-\beta_{2}\left(\eta_{3}-\eta\right) y_{f}}{\left(1-\eta_{3}\right) y_{f}}-\beta_{2}\right]+ \\
& +1\left(x-y_{f}\right) \frac{16}{15}\left(x-y_{f}\right)^{5 / 2}\left[B_{1}-\frac{C-H-\beta_{2}\left(\eta_{3}-\eta\right) y_{f}}{\left(1-\eta_{3}\right) y_{f}}\right]+ \\
& +\sum_{i=1}^{P-1} 1\left(x-\lambda_{1, i}\right) \frac{16}{15}\left(x-\lambda_{1, i}\right)^{5 / 2}\left(B_{i+1}-B_{i}\right)+1(x-\lambda) \frac{16}{15}(x-\lambda)^{5 / 2}\left[-\frac{D+C_{P}}{t}+B_{P}\right]+ \\
& +1(x-(\lambda+t)) \frac{16}{15}(x-(\lambda+t))^{5 / 2}\left[\frac{D+C_{P}}{t}+B_{P+1}\right]+ \\
& +\sum_{i=1}^{N-1} 1\left(x-\lambda_{2, i}\right) \frac{16}{15}\left(x-\lambda_{2, i}\right)^{5 / 2}\left(B_{P+i+1}-B_{P+i}\right)
\end{aligned}
$$

where $N$ and $P$ are the number of segments respectively before and after $x=\lambda$. No constraints are imposed on their values. This formulation also embeds the Rallabhandi formulation by taking: $N=2, P=1, \eta_{2}=0, \eta_{3}=\eta$. In addition if: $B_{1}=B_{2}=B_{3}, \eta=0.5, \xi=y_{f}, t=0$, it can be reduced to the Darden F-function. The new F-function model introduces new parameters to be defined by the designer on the basis of sole experience. To exploit the enriched parameterization, a strategy has to be defined in order to achieve low-boom signatures. Different metrics have been proposed throughout the years to evaluate the level of annoyance associated to a given sonic boom signature. The minimum front shock overpressure was one of the most employed criteria [44], but as shown in Makino and Kroo [45], this choice may introduce "low sonic boom signatures" with multiple shocks at very short intervals. Sullivan, using experimental tests on human response [46] suggested that the best predictor of loudness is the Stevens Mark VII perceived loudness metric also called PLdB [47]. This metric is adopted in this work to predict some of the unknown coefficients given by the retained F-function formulation (Eq. (5)) using an original optimization strategy that is developed in the following sections. 


\section{AIDA: Acoustic Inverse Design Approach}

\section{A. Evaluation of the F-function model coefficients}

A module called AIDA (Acoustic Inverse Design Approach) has been developed at ONERA to define low-boom configurations using the shaped sonic boom theory in the Python language. The main objectives of this module are threefold:

- the evaluation of the F-function coefficients defining its shape;

- the evaluation of the geometry area distribution starting from the corresponding equivalent area $A_{e}$;

- the definition of an F-function that minimizes a user-specified acoustic metric in the far-field.

The input data required are the flight conditions in terms of Mach number $M_{\infty}$, altitude $h$, aircraft weight $W$ and length $L$, desired front to rear pressure shocks ratio $P_{f} / P_{r}$, and the coefficients $B_{*}$, $\lambda_{*}$ and $y_{f}$. The length $L$ can be replaced by the front shock pressure rise $P_{f}$ depending on the procedure adopted for the evaluation of the coefficients (this aspect will be discussed later in this section).

To achieve the above first objective, some theoretical elements of geometrical acoustics are introduced. Propagation is performed using the nonlinear ray-tracing code TRAPS ( [48], [49]). The propagation code using as input the F-function solve the ray path equations of the disturbances emitted by the aircraft thus providing the signature at ground. The age parameter $\Lambda$ defines the steepening of the signal during propagation, as a result of nonlinear effects. The inverse of $\Lambda$ represents the slope $s$ of the area balance line (Fig. ??) defined as in [50]:

$$
s=-\frac{\sqrt{2 \beta}}{\gamma M_{h}^{3} \int_{0}^{h} \frac{p_{h}}{p} \sqrt{\frac{\rho a_{h}}{\rho_{h} a}} \sqrt{\frac{A_{h}}{z_{h} A}} \frac{M}{\beta} d z}
$$

where $A$ is the acoustic ray-tube area. This line is used to evaluate a front and a rear area balance in the F-function, thus obtaining the expression of two unknown coefficients $H$ and $C$. The front 
area balance is expressed as:

$$
\frac{1}{2} \Lambda C^{2}=\int_{0}^{y_{f}} F(x) d x
$$

If Eq. (9) is satisfied a shock is produced at location $y_{f}$ at an altitude $h$ below the aircraft. Using the definition of the F-function in Eq. (5) and Eq. (9), the parameter $H$ is written as:

$$
H=\frac{\frac{\Lambda C^{2}}{y_{f}}-\beta_{0} \eta_{2} \eta y_{f}-\beta_{2} y_{f}\left(\eta_{3}-\eta\right)^{2}+\left[C+\beta_{2}\left(\eta_{3}-\eta\right) y_{f}\right]\left(\eta_{3}-1\right)}{1-\eta-\eta_{2}+\eta_{3}}
$$

The rear shock area balance occuring between $L$ and $y_{r}$ is defined as:

$$
\int_{L}^{y_{r}} F(x) d x=\frac{1}{2}\left[F(L)+F\left(y_{r}\right)\right]\left(y_{r}-L\right)
$$

where $y_{r}$ is the total length of the signal, also obtained as the intersection between the area balance line starting from $F(L)$ and $F\left(y_{r}\right)$ :

$$
F\left(y_{r}\right)=F(L)+s\left(y_{r}-L\right)
$$

where $F(L)$ is evaluated using the expression defined in (5). Combining Eq. (12) and the constraints on the front and rear shock defined as:

$$
\frac{P_{f}}{P_{r}}=\frac{C}{F\left(y_{r}\right)-F(L)}
$$

yields :

$$
C=\frac{y_{r}-L}{\Lambda} \frac{P_{f}}{P_{r}}
$$

A typical value assumed for the shock ratio is 1 .

The remaining unknown coefficients require the introduction of additional equations on the rear part of the signal [39]. If a cylindrical wave is assumed, when $x>L F\left(y_{r}\right)$ is evaluated 
as:

$$
F\left(y_{r}\right)=-\frac{1}{\pi \sqrt{y_{r}-L}} \int_{0}^{L} \frac{\sqrt{L-x}}{y_{r}-x} F(x) d x
$$

The area under the $\mathbf{F}$-function between $L$ and $y_{r}$ can be expressed as:

$$
\int_{L}^{y_{r}} F(x) d x=-\frac{2}{\pi} \int_{0}^{L} F(x) \tan ^{-1} \sqrt{\frac{y_{r}-L}{L-x}}
$$

In order to evaluate $D$ it is necessary to use the analytical form of $A_{e}$ in Eq. (7) and its definition in Eq.(6). For the sake of simplicity, we define $\hat{D}$ as:

$$
\begin{aligned}
\hat{D}= & A_{e}(L, 0)-\frac{16}{15} \beta_{0} L^{5 / 2}-\frac{16}{15}\left(L-\eta_{2} y_{f}\right)^{5 / 2}\left[\frac{H-\beta_{0} \eta_{2} y_{f}}{\left(\eta-\eta_{2}\right) y_{f}}-\beta_{0}\right]-\frac{16}{15}\left(L-\eta y_{f}\right)^{5 / 2}\left[\beta_{2}-\right. \\
& \left.\frac{H-\beta_{0} \eta_{2} y_{f}}{\left(\eta-\eta_{2}\right) y_{f}}\right]-\frac{16}{15}\left(L-\eta_{3} y_{f}\right)^{5 / 2}\left[\frac{C-H-\beta_{2}\left(\eta_{3}-\eta\right) y_{f}}{\left(1-\eta_{3}\right) y_{f}}-\beta_{2}\right]+ \\
& -\frac{16}{15}\left(L-y_{f}\right)^{5 / 2}\left[B_{1}-\frac{C-H-\beta_{2}\left(\eta_{3}-\eta\right) y_{f}}{\left(1-\eta_{3}\right) y_{f}}\right]+ \\
& -\sum_{i=1}^{P-1} \frac{16}{15}\left(L-\lambda_{1, i}\right)^{5 / 2}\left(B_{i+1}-B_{i}\right)-\frac{16}{15}(L-\lambda)^{5 / 2}\left[-\frac{C_{P}}{t}+B_{P}\right]+ \\
& -\frac{16}{15}(L-(\lambda+t))^{5 / 2}\left[\frac{C_{P}}{t}+B_{P+1}\right]-\sum_{i=1}^{N-1} \frac{16}{15}\left(L-\lambda_{2, i}\right)^{5 / 2}\left(B_{P+i+1}-B_{P+i}\right)
\end{aligned}
$$

and thus obtaining

$$
D=\frac{\frac{15}{16} \hat{D} t}{(L-(\lambda+t))^{5 / 2}-(L-\lambda)^{5 / 2}}
$$

The $B_{i}$ are user defined parameters. When $B_{i}=0$ the maximum pressure is minimized; $0<B_{i}<s$ results in a minimized pressure rise signature while $B_{i} \geq s$ produces a $\mathrm{N}$-wave.

Two strategies can be adopted to find the unknown coefficients:

- Plotkin et al. [43] show that the equation set can be expressed as a system of two unknown parameters. As a consequence, the problem is reduced to two transcendental equations, solved analytically. This method avoids numerical errors that could be introduced while performing numerical integrals, but as a main drawback any change in the F-function parameterization requires a complete reformulation of the solution; 
- the equations can be solved numerically and the unknown coefficients evaluated as the result of an optimization loop. The solution becomes independent of the F-function model chosen.

The second choice is adopted here to solve the inverse design problem. Within AIDA, integrals are solved numerically with an adaptive Simpson method with tolerance set at $10^{-10}$. The minimisation problem is solved using an L-BFGS-B algorithm and using a termination criterion based on the projected gradient: the iteration is interrupted when $\max \left|P_{i}\right| i=1, \ldots, n<=10^{-5}$ where $P_{i}$ is the i-th component of the projected gradient and $n$ is the number of optimization variables. The cost function is defined as the absolute value of the difference between Eq. (16) left-hand-side and the right-hand-side terms which are evaluated separately.

Two different strategies are proposed depending on the set of design variables of interest: $\left(\lambda, y_{r}\right)$ or $(\lambda, L)$. The length of the aircraft is fixed by using the first design variable set, while the second one is useful if no constraints on the geometry are imposed and the designer wants to freeze the amplitude of the front shock. All the remaining unknown coefficients of the F-function are defined as functions of the two adopted design variables. If the couple $\left(\lambda, y_{r}\right)$ is adopted, the following process is proposed:

1. Assign user specified fixed parameters: $y_{f}$, flight conditions, aircraft weight, $P_{f} / P_{r}, B_{*}, L$, $t, \lambda_{1, *}, \lambda_{2, *}$. As already described the choice of the parameters $B_{*}$ defines the shape of the ground signature, while the values of the parameters $t, \lambda_{1, *}$ and $\lambda_{2, *}$ depends upon the designer experience. Section $C$ will provide a strategy for the definition of these parameters;

2. Evaluate $C$ from Eq. (14);

3. Evaluate $H$ from Eq. (10);

4. Determine the remaining unknowns using an optimization loop with a given initial guess for $\lambda$ and $y_{r}$ :

(a) Evaluate $D$ from Eq. (17) and Eq. (18);

(b) Evaluate $F\left(y_{r}\right)$ from Eq. (15); 
(c) Evaluate the right hand side of Eq. (16);

(d) Evaluate the left hand side of Eq. (16) from Eq. (11);

(e) Repeat the procedure until Eq. (16) is satisfied.

Slight modifications have to be introduced if the couple $(\lambda, L)$ is considered as design variables. Length $L$ is now a design variable and $C$ is evaluated as a function of the front shock pressure:

$$
C=\frac{p_{f}-p_{\infty}}{p_{\infty}} \frac{\sqrt{2 \beta r}}{\gamma M_{\infty}^{2}}
$$

Due to the local nature of the optimization algorithm it appears that by changing the starting point, it is possible to obtain different combinations of the two design variables that could satisfy (16).

\section{Validation test case}

The validation test case is based on the work of Darden [39], adopted as validation also in [40] by Rallabhandi and Mavris. The input values are given in Table 1. AIDA is run considering both the variable couple $\left(\lambda, y_{r}\right)$ with a fixed $L$ as in [39] and [40], and the modified version fixing the value of the target bow shock to $P_{f}=50.86 P a$. In the latter case the length obtained at convergence is $L=91.60 \mathrm{~m}$. The results obtained with AIDA (Fig. 3) show a mean relative error of less than $2.5 \%$ compared to the ones obtained in the literature as shown in Table 2.

\begin{tabular}{|lr|}
\hline \hline Variable & Value \\
\hline$M$ & 2.7 \\
$L$ & $91.46 \mathrm{~m}$ \\
$W$ & $272,155.42 \mathrm{~kg}$ \\
$B$ & $0 \mathrm{~m}^{-0.5}$ \\
$y_{f}$ & $9.146 \mathrm{~m}$ \\
\hline \hline
\end{tabular}

Table 1. Input values for AIDA validation test case.

\section{B. Evaluation of the geometrical area distribution}

AIDA provides the area distribution of an equivalent axi-symmetric body that corresponds to the desired F-function. However, the formulation of the inverse problem does not guarantee the 


\begin{tabular}{|c|cccc|}
\hline \hline Variable & Darden [39] & Rallabhandi [40] & AIDA(fixed $L$ ) & AIDA (fixed $P_{f}$ ) \\
\hline$H\left[m^{0.5}\right]$ & 0.191 & 0.194 & 0.192 & 0.192 \\
$C\left[m^{0.5}\right]$ & 0.0303 & 0.0305 & 0.0304 & 0.0304 \\
$D\left[m^{0.5}\right]$ & 0.0377 & 0.0373 & 0.0409 & 0.0406 \\
$\lambda[m]$ & 82.514 & 81.743 & 82.603 & 82.485 \\
$y_{r}[\mathrm{~m}]$ & 153.627 & 154.027 & 153.598 & 153.747 \\
\hline \hline
\end{tabular}

Table 2. Comparison of the AIDA analysis with previous works.

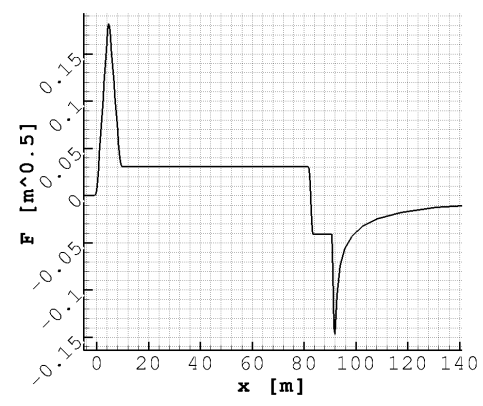

a) F-function.

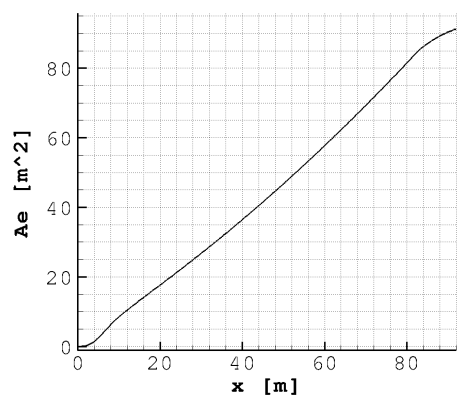

b) Equivalent area.

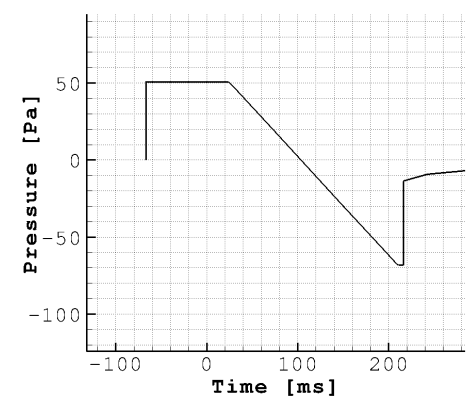

c) Ground signature.

Figure 3. Validation test case results.

uniqueness of the geometrical area distribution that corresponds to the equivalent area distribution. In fact, if we assume that for a wing-body configuration, the $A_{e}$ distribution can be decomposed as follows:

$$
A_{e}=A_{v}^{\text {fuse }}+A_{v}^{\text {wing }}+A_{l}^{\text {fuse }}+A_{l}^{\text {wing }}
$$

different combinations of these terms can produce the same result. In addition, acting on the geometry impacts explicitly the volume terms but has also an indirect influence on the lift terms, in particular when the wing shape is modified. The choice of fuselage tailoring, based on direct optimization with the Nealder - Mead simplex algorithm, has been made to achieve the target shaped configuration to limit the impact of shape modification on the lift contribution. Not include the wing in the design has as main consequence to amplify the modifications of the fuselage volume that in some cases are not allowed. The problem can be stated as a minimization problem of a cost function that describes the error between the actual and the target designed configurations. Design variables are radius values at several locations along the fuselage axis. Using these geometrical parameters, the 3D structured mesh is generated automatically and an Euler CFD computation 
is performed using the ONERA elsA [51] solver to evaluate the lift terms. The Roe flux scheme with the Harten entropy correction and the Van Albada flux limiter are adopted as numerical parameters. The computation terminates when a tolerance of $10^{-6}$ on the aerodynamic coefficents is satisfied or when the maximum number of iterations is achieved (500 iterations in this case). The typical mesh and the grid topology adopted are shown in Fig. 4.
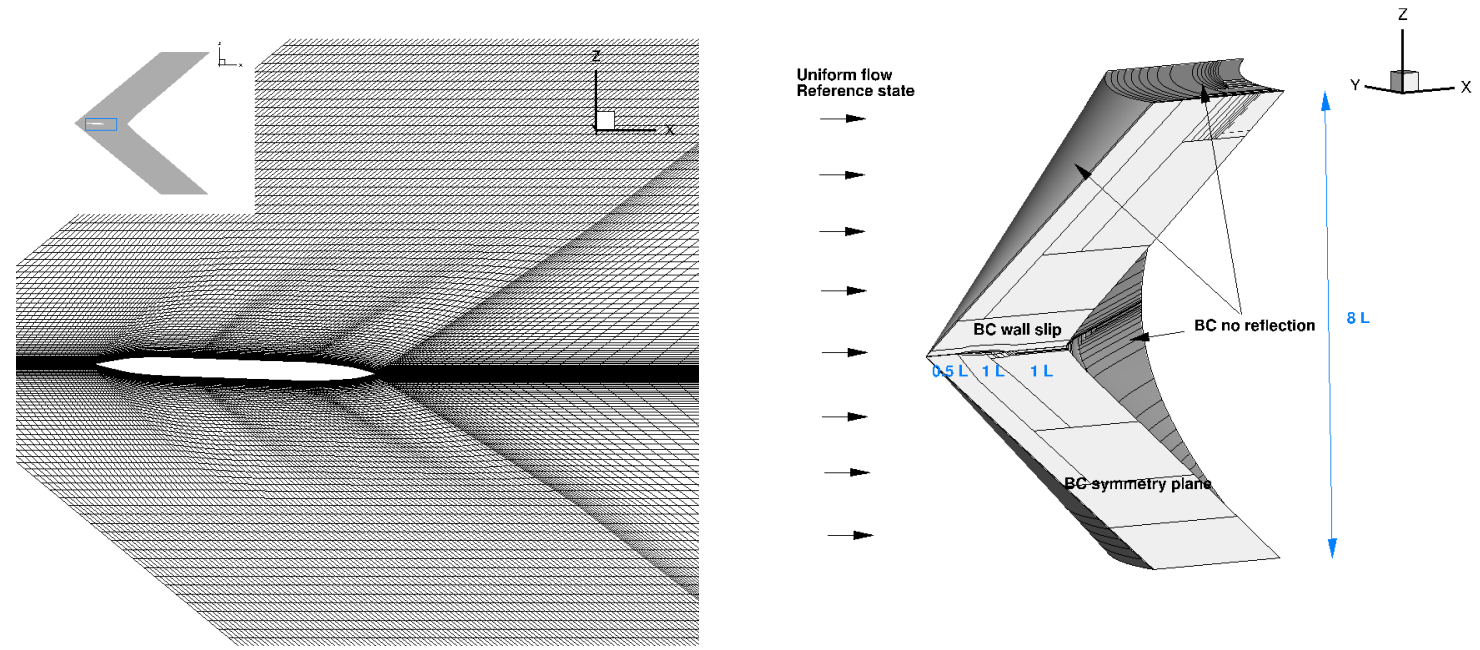

Figure 4. Symmetry plane mesh and domain boundary conditions (BC) adopted for the elsA computations.

Using the surface skin mesh and the corresponding pressure field solution, the equivalent area distribution and the F-function are evaluated using an in-house post-processing module. The volume term of the area distribution is evaluated directly on the 3D geometry mesh, while local lift contribution of each section that defines the geometry is the result of the numerical integration of the pressure coefficient over the section countour. Once the equivalent area distribution is defined, the F-function is evaluated using finite differences and numerical integration. The fuselage tailoring is a critical and fundamental aspect of the inverse design, as several factors have an impact on the final solution:

- the parameterization of the fuselage (number of DVs, control point distribution, kind of parametric curve,...);

- the objective cost function;

- the lift distribution evaluation. 


\section{Impact of geometry parameterization}

Different aspects are fundamentals in the description of the fuselage geometry also in our simple case where the fuselage is axi-symmetric and its shape defined using a revolution along the main axis of a control line. Among them we can include: the nature of the parametric curve adopted, the number of design variables considered as well as their distribution. The choice of the parametric curve can hardly be defined a priori. Two approaches have been considered: Bézier curve and cubic spline. A geometry obtained using Bézier curves is described by control points which do not belong to the real geometry, thus helping in the description of complex smooth geometries but limiting the control on local curvature. In addition the Whitham function is very sensitive to $A_{e}^{\prime \prime}$ [30]. For these two reasons, the cubic spline has finally been chosen in this study to describe the fuselage geometry.

The choice of the design variables is also critical, and a compromise has to be achieved between a fine and a coarse parameterization. A fine parameterization is able to better describe the geometry, but can slow down significantly the shape optimization convergence, due to the increased design search space. On the opposite a coarse parameterization is unable to produce modifications of the second derivative that create a low-boom compliant F-function. A coarse (10 control points) and a fine (27 control points) parameterization, respectively named $\mathrm{A}$ and $\mathrm{B}$, are considered on a non-lifting axi-symmetric body at an altitude of 18,288 $\mathrm{m}$ and a Mach number $M_{\infty}=1.6$. The points are uniformly distributed along the fuselage axis and can move in any direction of the $\mathrm{xz}$ plan with the exception of the nose and rear tips that are fixed. The objective is to shape the configuration to obtain a flat-top ground signature. The design parameters obtained using the $\left(\lambda, y_{r}\right)$ couple approach are defined in Tables ?? and 4 and shown in Fig. 5.

\begin{tabular}{|cc||cc|}
\hline \hline Var. & Value & Var. & Value \\
\hline$W$ & $0 \mathrm{~kg}$ & $t$ & $0 \mathrm{~m}$ \\
$N, P$ & 1 & $\eta_{2}$ & 0 \\
$B_{1}, B_{2}$ & $0 \mathrm{~m}^{-0.5}$ & $\eta_{3}$ & $\eta$ \\
$\eta$ & 0.5 & $y_{f}$ & $9.84 \mathrm{~m}$ \\
$\beta_{0}, \beta_{2}$ & $0 \mathrm{~m}^{-0.5}$ & $L$ & $30 \mathrm{~m}$ \\
\hline \hline
\end{tabular}

Table 3. AIDA input coefficients.

\begin{tabular}{|cc|}
\hline \hline Var. & Value \\
\hline$H$ & $0.00909 m^{0.5}$ \\
$C$ & $0.00270 m^{0.5}$ \\
$D$ & $0.0154 m^{0.5}$ \\
$y_{r}$ & $33.537 m$ \\
$\lambda$ & $21.341 m$ \\
\hline \hline
\end{tabular}

Table 4. Coefficients evaluated with AIDA. 


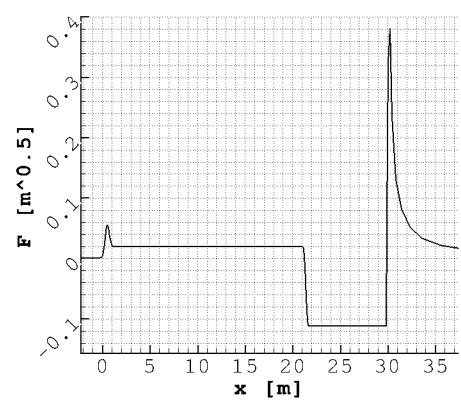

a) F-function.

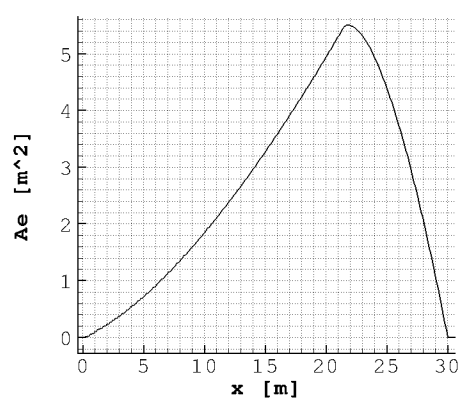

b) Equivalent area.

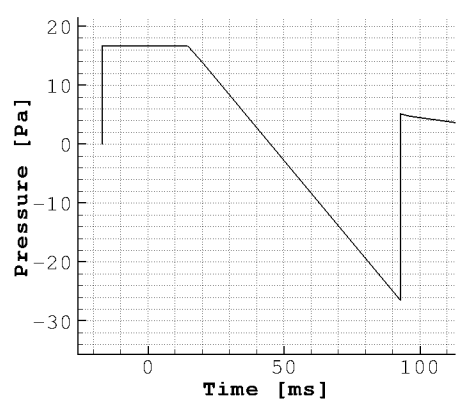

c) Ground signature.

Figure 5. Minimum overpressure solution obtained using AIDA for the fuselage non-lifting configuration.

Due to the fact that a non-lifting configuration is considered, the weight is set to $W=0$ in order to satisfy the condition $A_{e}(L)=A_{v}^{f u s e}(L)=0$. The fuselage being considered as a slender body, a good initial solution is obtained by approximating at first order the geometrical area distribution $\hat{A}_{g}$ with the equivalent area, so that $\hat{A}_{g}(x)=A_{e}(x)$. The ability for the chosen parameterization to fit the geometrical target area distribution is evaluated comparing the absolute errors resulting from the approximation by cubic spline of the initial area distribution $\hat{A}_{g}$. Figures 6 and 7 show the geometrical and the curvature errors respectively for parameterization A and B. Significant errors can be noticed where $F^{\prime}$ changes its sign: at the nose and rear region, as well as at $x=\lambda=21.34$ $m$. In these critical regions, multiple variations of the sign of $F^{\prime}$ occur therefore impacting the curvature of the corresponding $A_{e}$ distribution. Notice that in weak curvature variation regions, parameterization B provides a more accurate geometry. The comparison between the configuration obtained with the two initial solutions is shown in figure 8. It is notable on the one hand, that parameterization A shows oscillations in the first half of the F-function that result in a multi-shock ground signature response. On the other hand, parameterization B provides a satisfactory detailed description of the configuration as it allows to reproduce the desired flat-top ground signature. Considering these results, parameterization B is retained for the following studied cases. 


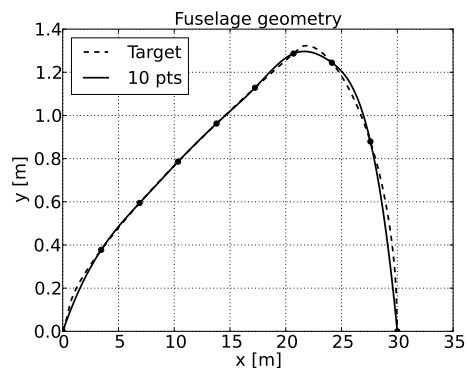

a)

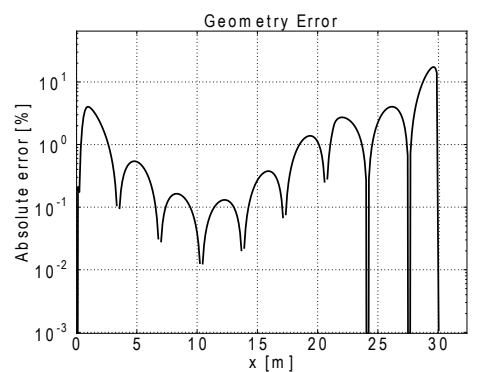

b)

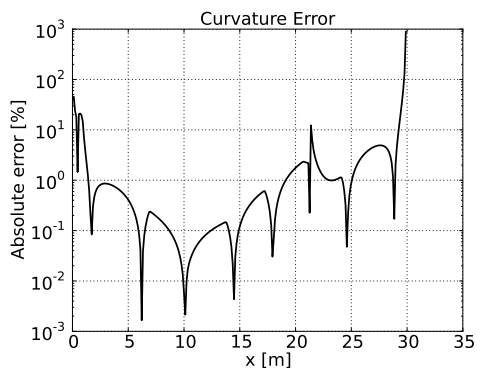

c)

Figure 6. Initial geometry with 10 points uniformly distributed. (Param. A).

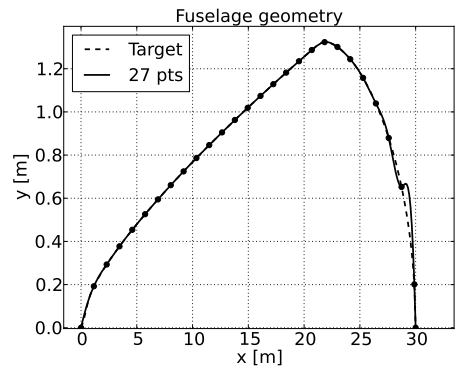

a)

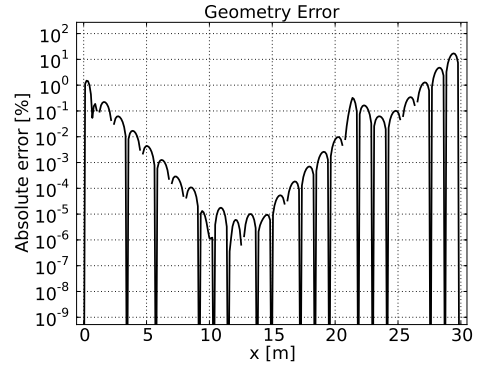

b)

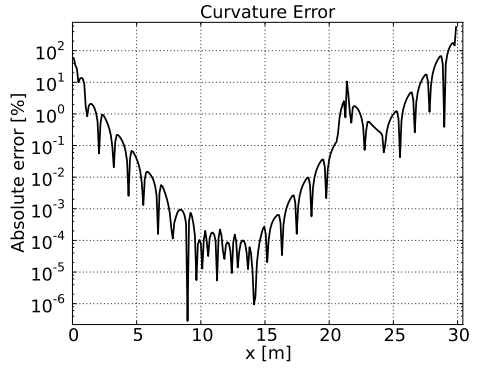

c)

Figure 7. Initial geometry with 27 points uniformly distributed. (Param. B).

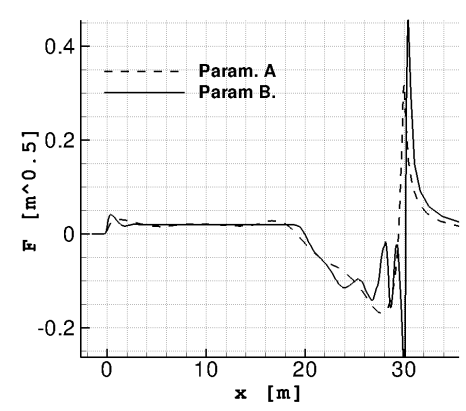

a) F-function.

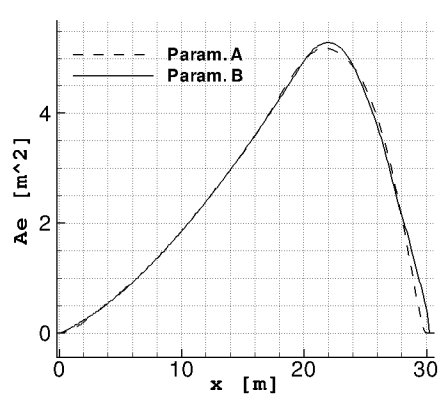

b) Equivalent area.

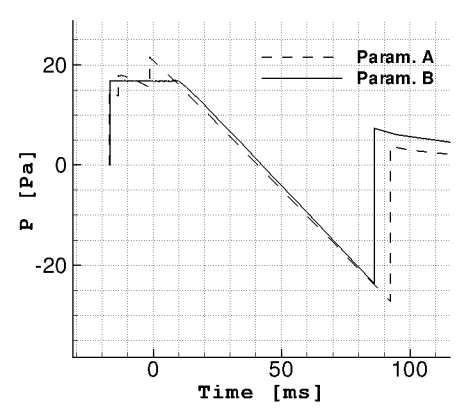

c) Ground signature.

Figure 8. Configuration obtained using the two initial approximated geometries. 


\section{Impact of objective cost function formulation}

The objective function chosen to tailor the geometry has been defined in the present approach as an integral error estimator which general formulation is:

$$
J_{f}=\int_{0}^{L}\left|f(x)-f^{\text {target }}(x)\right| d x
$$

Three functions $f(x)$ have been considered in the present study: $A_{e}(x), F(x)$ or $\Delta p(x)$. The intent is to analyze the impact of the cost function on the final solution as well as the optimizer behaviour to select the most suitable function for future studies. To analyze the impact of each cost function on the final configuration, the initial solution obtained with the fine parameterization is perturbed by the introduction of two bumps in the vicinity of the nose. This is due to the fact that, as previously explained, the initial solution is a good approximation of the targeted configuration (see Fig. 8). Figures (9-13) shown the comparison between the different objective functions presented in Eq. (21). The optimization based on $J_{A_{e}}$ shows a good matching between the optimized and the target $A_{e}$ (Fig. 10a) as a result of a decrease of the objective by more than $80 \%$ (Fig.13a). The corresponding F-function (Fig. 9a) shows some oscillations at the $F^{\prime}$ discontinuity located around $\lambda$. The resulting ground signature (Fig. 11a) shows multiple shocks instead of a flat-top. The configuration obtained using $J_{F}$ as objective results in a Whitham F-function (Fig. 9b) with less oscillations than those obtained with the other two strategies. The $A_{e}$ distribution is different from the target, but it remains smooth and reduces the initial bumps, which has a positive impact on the resulting geometry (Fig. 12b) in terms of curvature fluctuation reduction. The use of the ground signature as cost function $J_{P}$ did not result in the elimination of the initial oscillations of the geometry (Fig. 12c). The corresponding F-function (Fig. 9c) shows two peaks after the first thus producing a ground signature (Fig. 11c) close to the initial one. Furthermore, two shocks still persist in the bow region. The analysis shows that the $A_{e}$ and the F-function based optimizations

provide complementary informations required to obtain the desired shaped configuration. As a result of this preliminary study, the choice has been oriented toward the definition of a cost function 


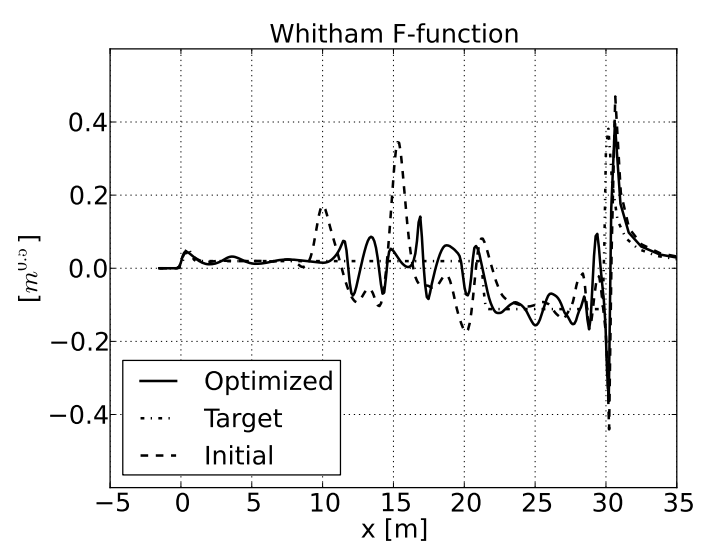

a) $A_{e}$ based optimization.

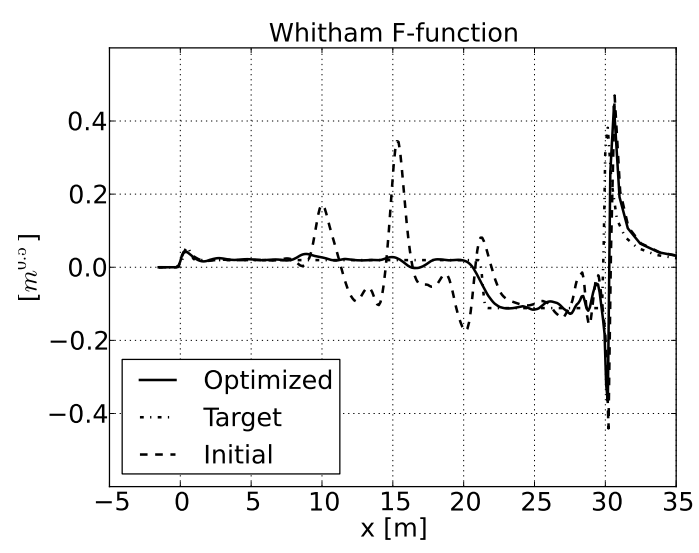

b) F-function based optimization.

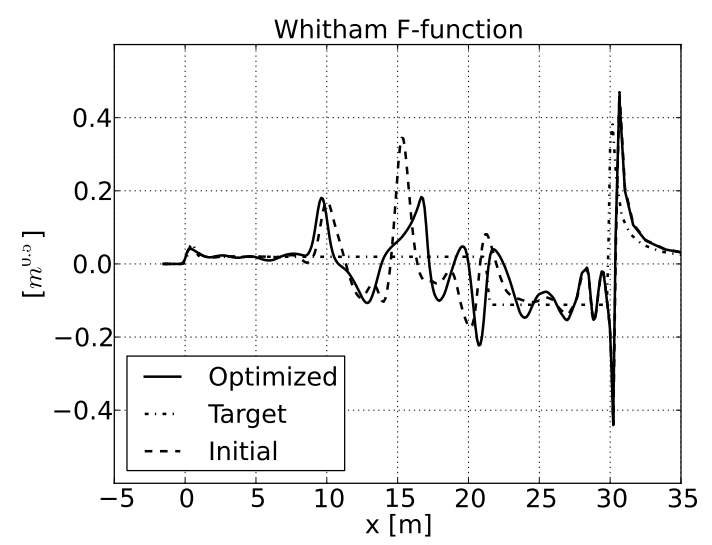

c) Signature based optimization.

Figure 9. Whitham F-function obtained with the different objectives - Parametrization B: 27 design variables.

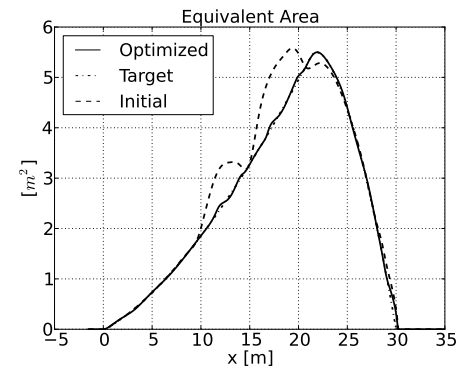

a) $A_{e}$ based optimization.

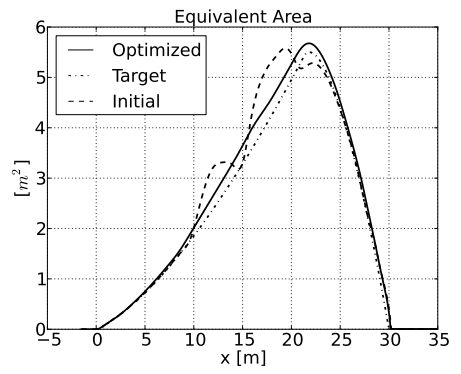

b) F-function based optimization.

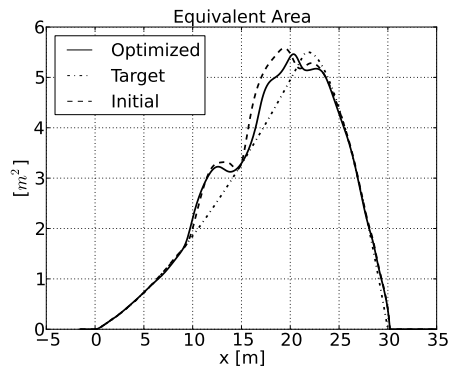

c) Signature based optimization.

Figure 10. Equivalent area obtained with the different objectives - Parametrization B: 27 design variables. 


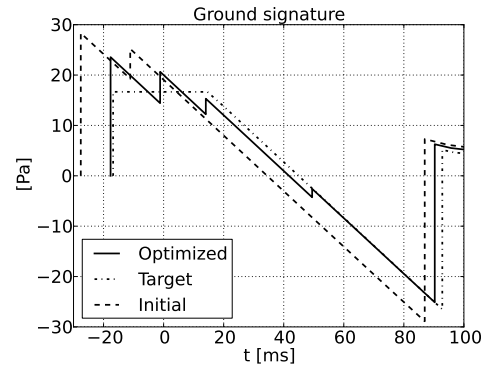

a) $A_{e}$ based optimization.

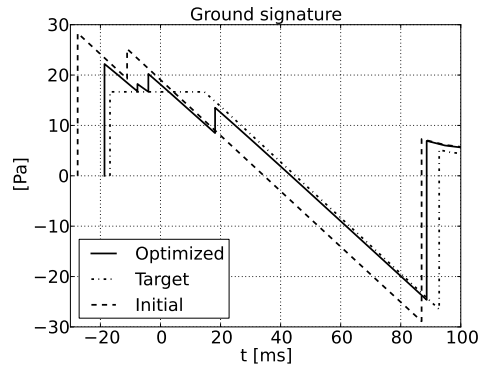

b) F-function based optimization.

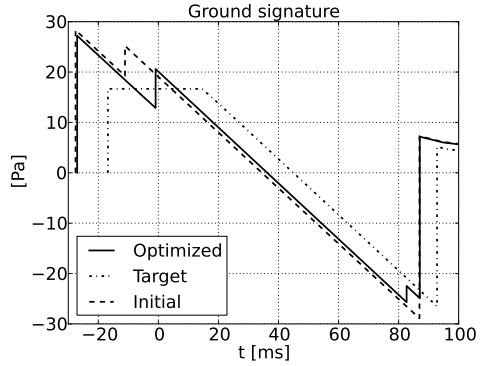

c) Signature based optimization.

Figure 11. Ground signature obtained with the different objectives - Parametrization B: 27 design variables.

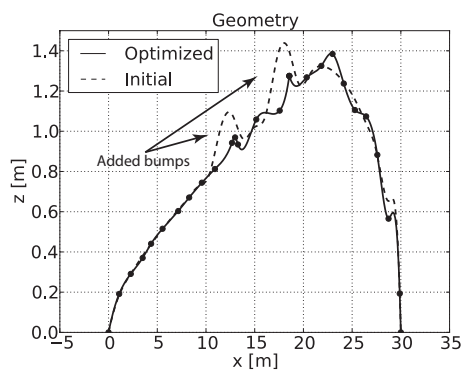

a) $A_{e}$ based optimization.

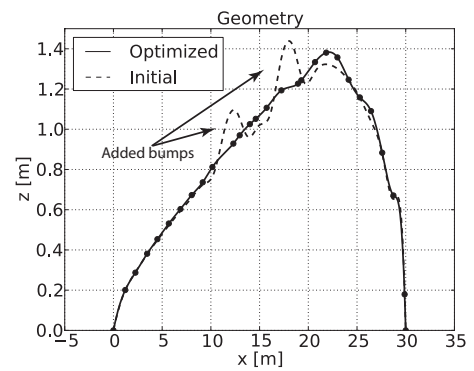

b) F-function based optimization.

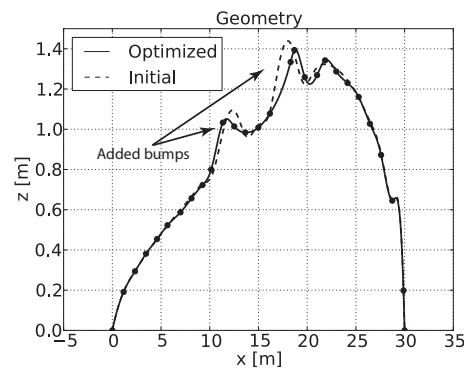

c) Signature based optimization.

Figure 12. Radius distribution obtained with the different objectives - Parametrization B: 27 design variables.

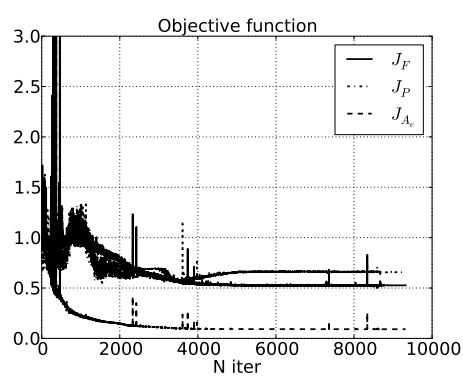

a) $A_{e}$ based optimization.

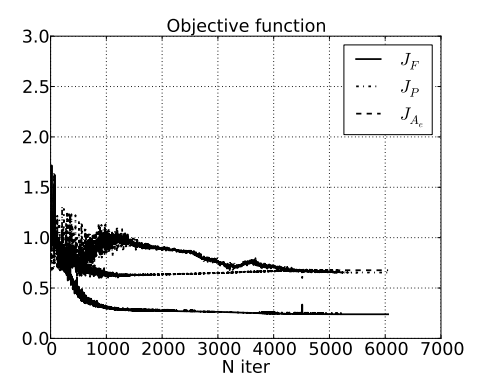

b) F-function based optimization.

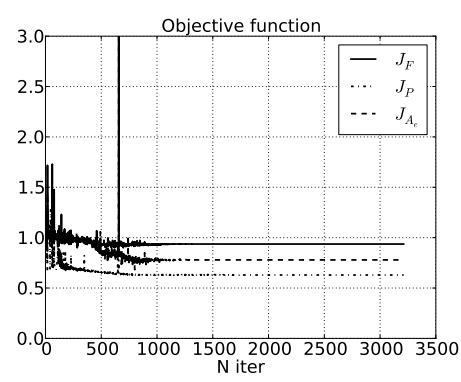

c) Signature based optimization.

Figure 13. Objectives normalized with respect to their respective initial value. 
as a linear combinations of these two cost functions:

$$
J_{\text {comb }}=\frac{J_{A_{e}}}{J_{A_{e}}^{0}}+\frac{J_{F}}{J_{F}^{0}}
$$

where each objective function is normalized with the value at the first iteration of the optimization process $J_{(.)}^{0}$. Using as objective $J_{c o m b}$, the resulting Whitham F-function (14a) shows an approx-

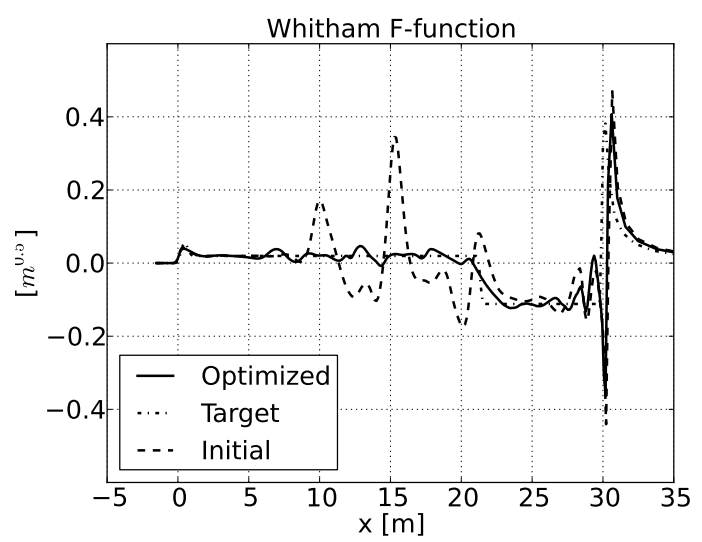

a)

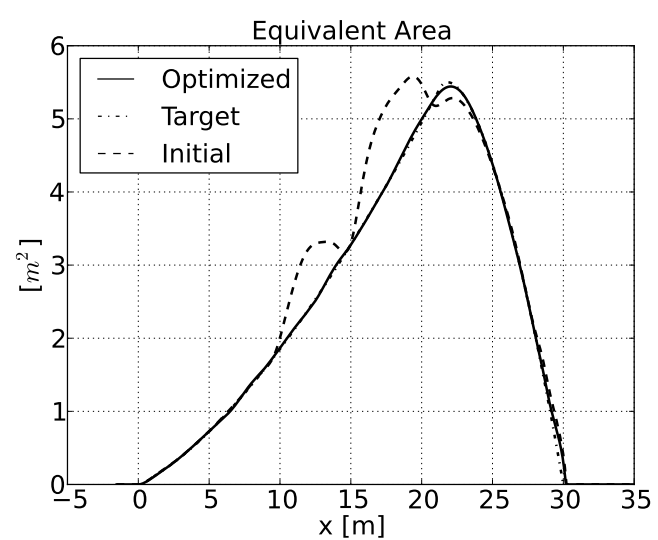

b)

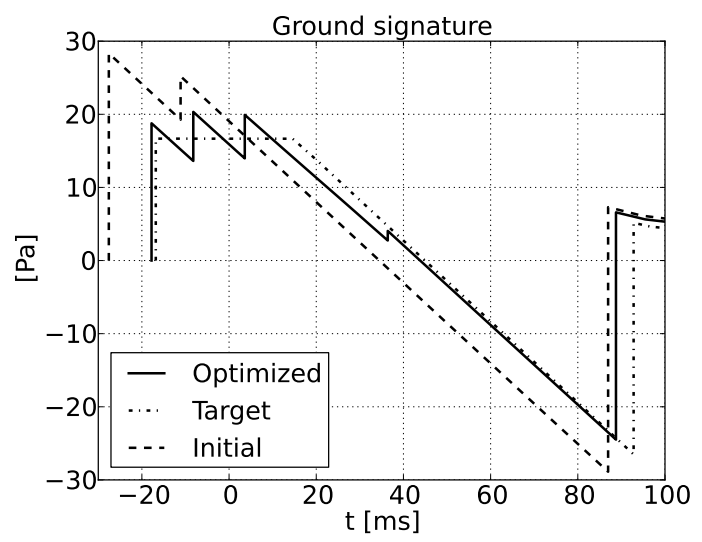

c)

Figure 14. Optimization based on linear combination of objective functions using 27 variables.

imation of the target without the oscillations, observed using $J_{F}$, for $x \leq \lambda$. In addition the zero slope achieved after the initial peak and the limited angle variation at $F(\lambda)$ allow to reach a multishock approximation of the flat-top ground signature (Fig. 14c). The oscillations in the second half of the signal have a limited extent around the discontinuity in $x=L$ resulting in a negligible impact on the ground signature. The equivalent area (Fig. 14b) approximates the optimal target reducing 


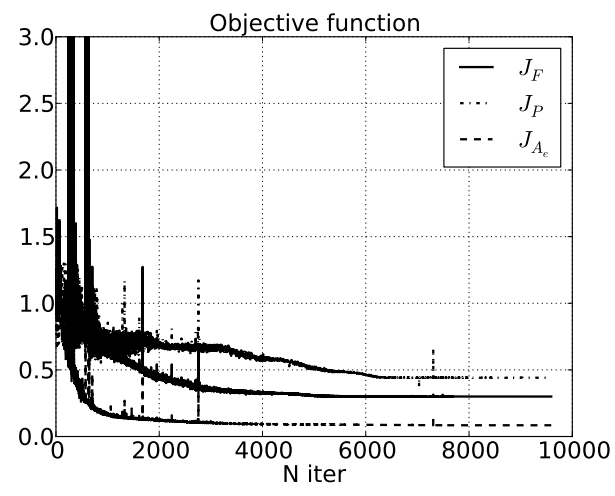

a)

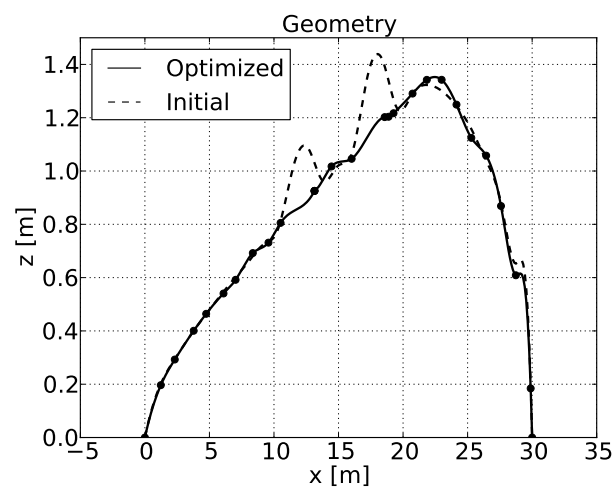

b)

Figure 15. Optimization based on linear combination of objective functions using 27 variables.

$J_{A_{e}}$ (see Fig. 15 a) by more than $80 \%$ w.r.t. the initial geometry. Each objective component $J_{A_{e}}$, $J_{F}$ and $J_{P}$ is reduced using $J_{\text {comb }}$ as objective, producing an accurate approximation of the target configuration.

\section{Definition of Low-Boom F-function}

The shaped sonic boom theory does not take into account the acoustic metrics currently used to evaluate people annoyance due to sonic boom (e.g. PLdB, dBA,..) [52]. The generalisation of the F-function parameterization have introduced additional coefficients that have to be defined by the designers. These coefficients can be exploited to define a low-PLdB F-function. The strategy proposed consists in coupling the AIDA module and an external optimization loop as shown in Fig. 16. The design variables are the AIDA input parameters that defines the F-function $\left(B_{*}, \eta_{*}, t, y_{f}\right)$. At each optimization iteration AIDA evaluates the remaining unknown coefficients $\left(H, C, D, \lambda, y_{r}\right)$, thus defining the $A_{e}$ distribution, the F-function and from there the corresponding ground signature can be calculated using the propagation code TRAPS.

The optimization is performed using GADO [53], a genetic algorithm integrated to the Dakota optimization library [54]. To evaluate PLdB, the zero-thickness signal is post-processed introducing the rise time as function of the peak overpressure. The idea is based on the theoretical model developed by Kang and Pierce [55]:

$$
\tau_{0.1-0.9}=c / \Delta p
$$




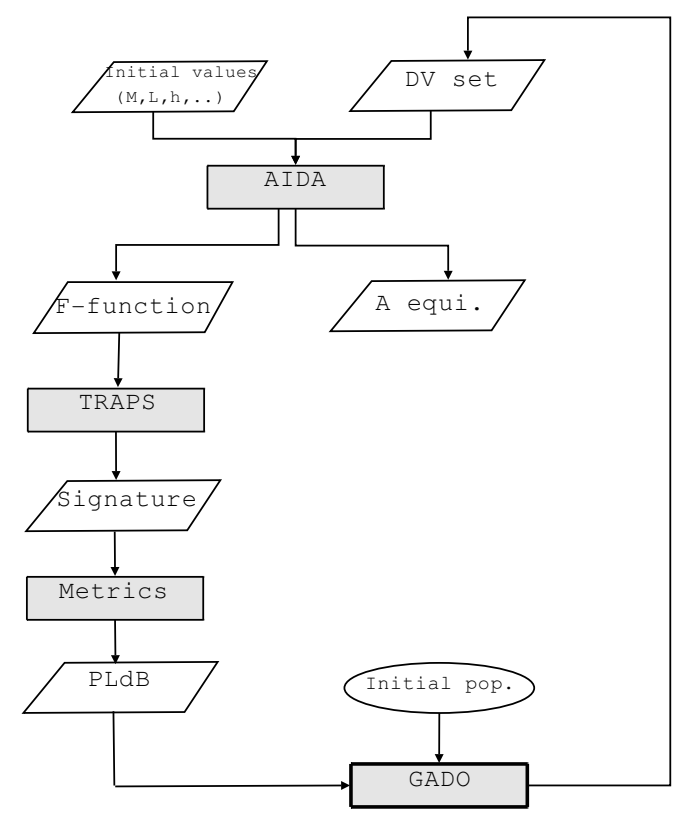

Figure 16. Flow-chart of the coupling between AIDA and the optimizer.

where $c$ is an empirical coefficient strongly affected by the atmosphere humidity. In this study $c$ is taken equal to $20.88 \times 10^{-} 3 \mathrm{~Pa} \mathrm{~ms}^{-1}$. Rise time is modeled applying an hyperbolic tangent to the signature as in [56]:

$$
p(t)_{r t}=\frac{\Delta p(t)}{2}\left[1+\tanh \left(c_{r} \frac{t}{\tau_{0.1-0.9}}\right)\right]
$$

where $c_{r}$ is a coefficient equal to 1.472 for the rise time definition (including $10 \%$ to $90 \%$ of the response signal peak overpressure). In Table 5 are shown the lower and upper bounds of the design variables. The other input required to solve the problem are shown in Table 6. The

\begin{tabular}{|cc|cc|}
\hline \hline & Variable & Lower bound & Upper bound \\
\hline$B_{i} \quad \forall i \in[1, N+P]\left[m^{-0.5}\right]$ & $-9.15 \times 10^{-5}$ & $2.44 \times 10^{-4}$ \\
& $\beta_{0}\left[m^{-0.5}\right]$ & $3.05 \times 10^{-6}$ & 0.03 \\
$\beta_{2}\left[m^{-0.5}\right]$ & -0.03 & 0.03 \\
& $t[m]$ & 0 & 1.524 \\
$\eta$ & 0.2 & 0.8 \\
$y_{f}[m]$ & 1.524 & 4.573 \\
\hline \hline
\end{tabular}

Table 5. Ranges for F-function optimization.

optimized coefficients (Table 7) and the other coefficients evaluated by AIDA (Table 8) correspond to a configuration that creates a 88.84 PLdB ground signature. The optimizer reduces of nearby 2 PLdB the initial value determining a low-PLdB solution shown in Fig. 18. The initial peak 


\begin{tabular}{|c|c|c|c|}
\hline Constant & Value & Constant & Value \\
\hline$M$ & 1.6 & $\lambda_{1,1}$ & $y_{f}+9.146$ \\
\hline$L$ & $36.58 \mathrm{~m}$ & $\lambda_{2,1}$ & $\lambda+t+1.524$ \\
\hline$W$ & $45,000 \mathrm{~kg}$ & $\forall i \in[2, N]$ & $\lambda_{1, i-1}+1.524$ \\
\hline $\begin{array}{c}N, P \\
h\end{array}$ & $\begin{array}{c}3 \\
18,000 \mathrm{~m}\end{array}$ & $\forall i \in[2, P]$ & $\lambda_{2, i-1}+1.524$ \\
\hline
\end{tabular}

Table 6. Additional fixed input values.

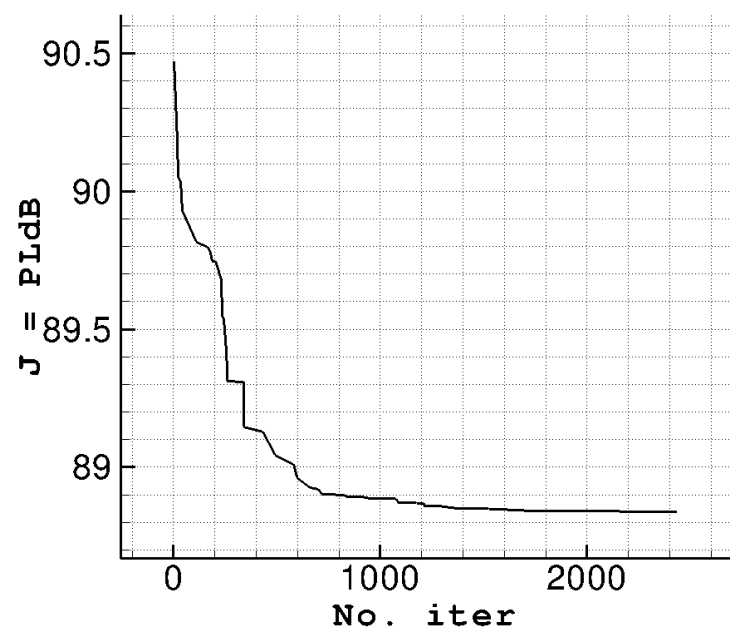

Figure 17. F-function input coefficient optimization history.

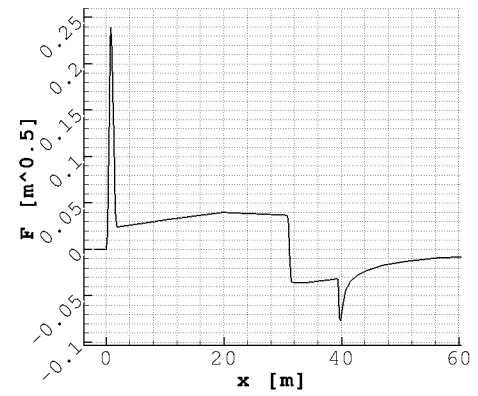

a) F-function.

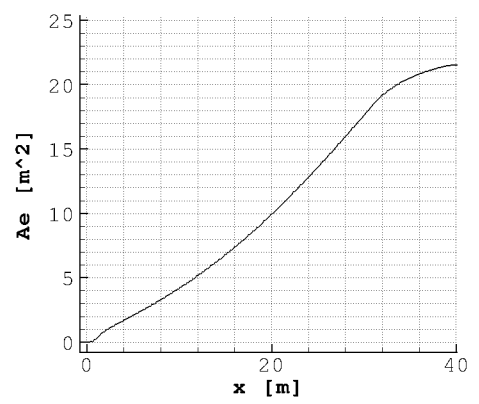

b) Equivalent area.

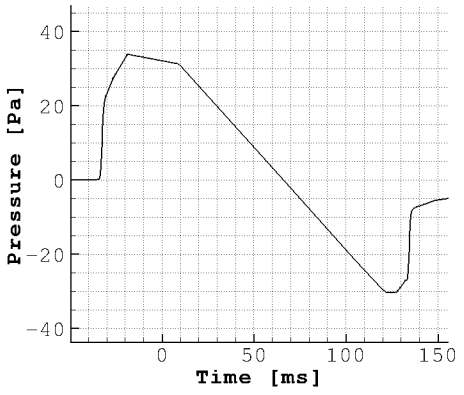

c) Ground signature.

Figure 18. Low-PLdB configuration.

of the F-function tends to a $\delta$ Dirac aiming at reducing the coefficient $C$ and so the front shock amplitude. The first shock is then followed by a ramp-like behaviour and a subsequent expansion. The same behaviour occurs in the rear part of the acoustic signal. The result shows that the ground signature tends to become a sinusoidal-like shock-free signal. Nevertheless it could be applied to 


\begin{tabular}{|cc||cc|}
\hline \hline Variable & Value & Variable & Value \\
\hline$B_{1}\left[m^{-0.5}\right]$ & $9.426 \times 10^{-4}$ & $B_{4}\left[m^{-0.5}\right]$ & $-8.313 \times 10^{-5}$ \\
$B_{2}\left[m^{-0.5}\right]$ & $8.237 \times 10^{-4}$ & $B_{5}\left[m^{-0.5}\right]$ & $-2.660 \times 10^{-5}$ \\
$B_{3}\left[m^{-0.5}\right]$ & $-2.798 \times 10^{-4}$ & $B_{6}\left[m^{-0.5}\right]$ & $7.416 \times 10^{-4}$ \\
$\eta$ & 0.415 & $y_{f}[\mathrm{~m}]$ & 1.524 \\
$\beta_{0}\left[m^{-0.5}\right]$ & 0.00498 & $t[m]$ & 0.0643 \\
$\beta_{2}\left[m^{-0.5}\right]$ & -0.180 & & \\
\hline \hline
\end{tabular}

Table 7. Optimized value of the coefficients.

\begin{tabular}{|cc|}
\hline \hline Variable & Value \\
\hline$H\left[m^{0.5}\right]$ & $2.874 \times 10^{-4}$ \\
$C\left[m^{0.5}\right]$ & $-2.511 \times 10^{-4}$ \\
$D\left[m^{0.5}\right]$ & $-8.531 \times 10^{-5}$ \\
$y_{r}[\mathrm{~m}]$ & 48.216 \\
$\lambda[\mathrm{m}]$ & 25.736 \\
\hline \hline
\end{tabular}

Table 8. Coefficients evaluated with AIDA.

any problem in which it is required to define a target F-function.

\section{Wing-body configuration design}

\section{A. Non lifting fuselage tailoring}

Considering a non-lifting wing-body configuration with non cambered symmetric cross-sectional wing at $A o A=0^{\circ}$, Eq.(20) reduces to: $A_{e}=A_{v}^{\text {fuse }}+A_{v}^{\text {wing }}$. In an attempt to retrieve the target area distribution, the fuselage is tailored while the wing shape is frozen. The initial wing-body

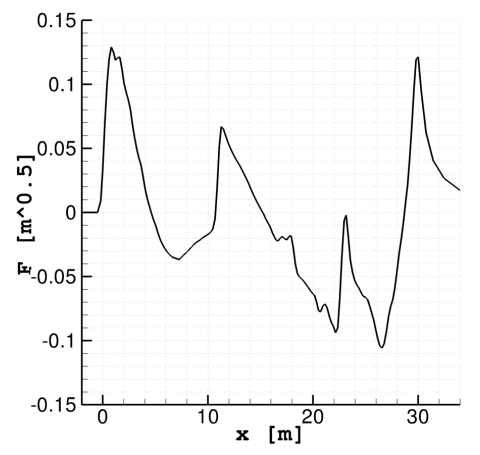

a) F-function

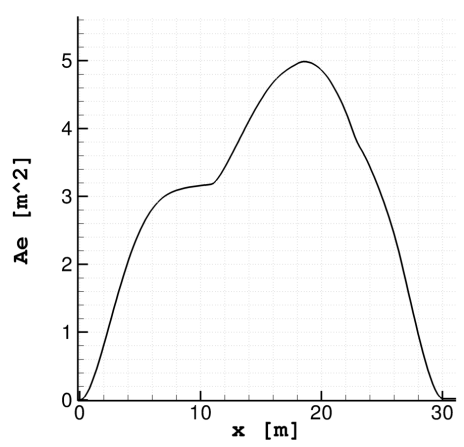

b) Equivalent area

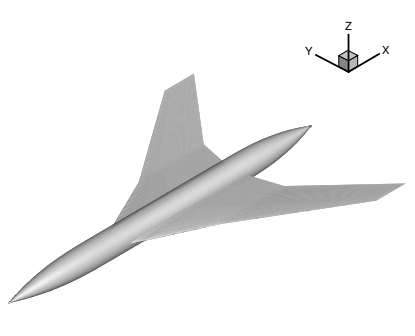

c) Geometry

Figure 19. Wing-body initial configuration $\left(A o A=0^{\circ}\right)$.

configuration F-function, $A_{e}$ distribution and geometry are shown in Fig. 19. The target 
shaped configuration is the same as the one defined in Tables 3-4 and is plotted in Fig.20. The initial approximated geometrical area distribution of the fuselage is obtained by cancelling the wing volume contribution from the total area distribution :

$$
\hat{A}_{g}^{\text {fuse }}(x) \simeq A_{v}^{\text {fuse }}(x)=A_{e}(x)-A_{v}^{\text {wing }}(x)
$$

The design variable set selected consists in 41 radius of fuselage section equally distributed along

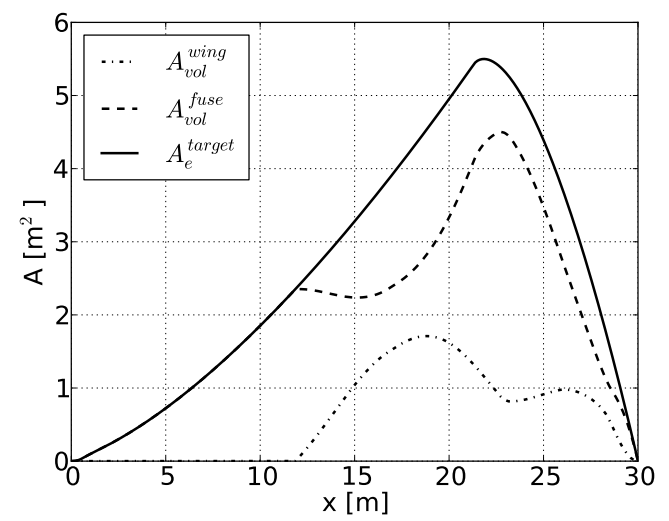

Figure 20. Initial tailoring of the fuselage area distribution.

the fuselage axis: 13 for the nose region, 15 for the cabin and 13 for the aft part. An optimization approach as previously described based on the composite objective function defined in Eq. (21) results in a cost function reduction around $75 \%$ of its initial value. The resulting $A_{e}$ distribution (Fig. 21b) is in excellent agreement with the target.

The F-function (21a) obtained after the tailoring shows reduced oscillations around the target function, especially in the first part of the signal $(x<20) \mathrm{m}$. As a direct consequence the ground signature (21c) results in an almost overlapping flat-top pattern. The oscillations in the aft part of the F-function still persist as in the fuselage alone design case analyzed previously, but they produce a minor impact on the ground signature.

The strategy proposed alleviates the oscillations of the F-function related to the discontinuities of the geometry, like the wing in the present case, and can be applied without modification to other discontinuities such as engine nacelle or lifting surfaces. Figure $22 \mathrm{~b}$ shows the geometry obtained after less than 6,000 fuselage tailoring iterations (Fig. 22a). 


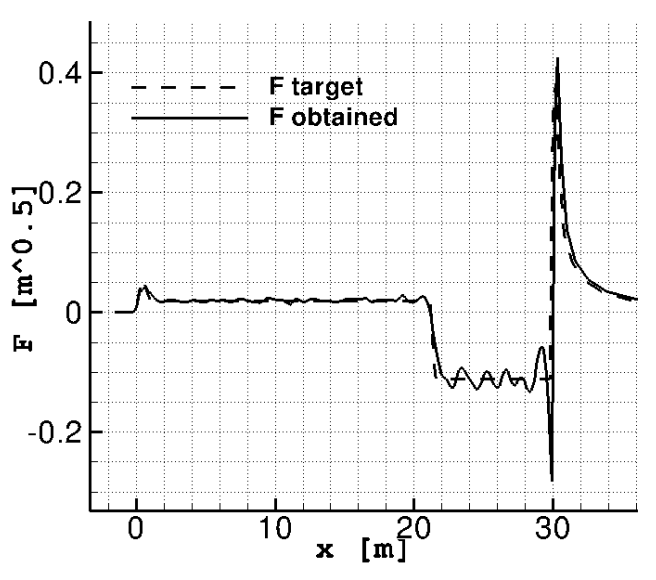

a) F-function

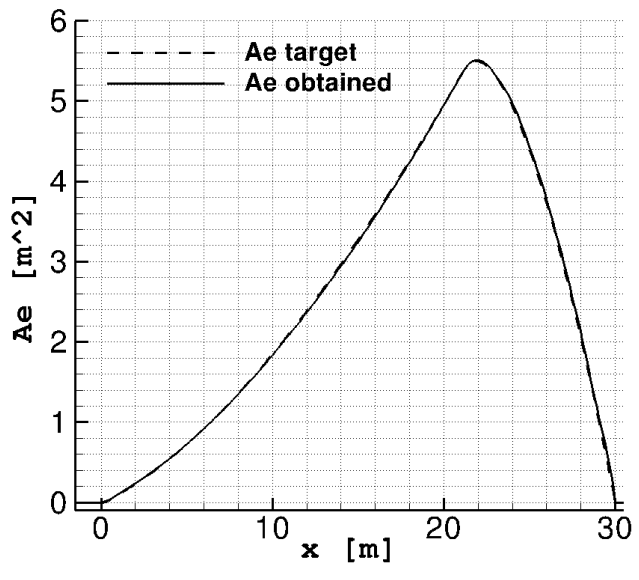

b) Equivalent area

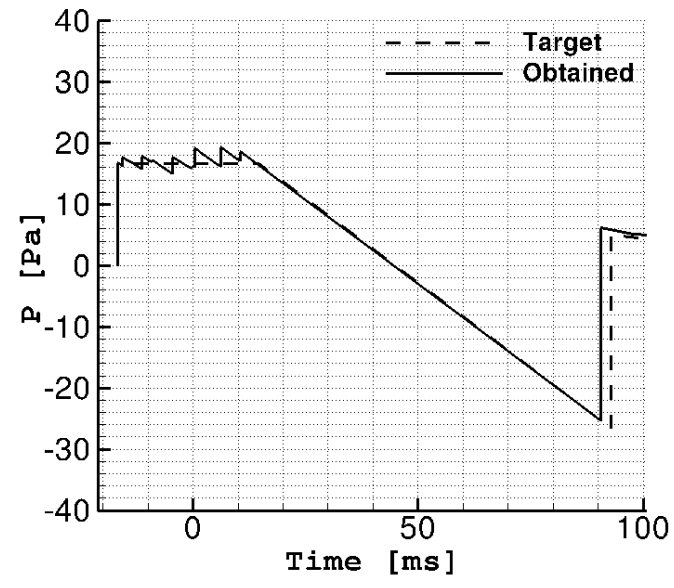

c) Ground signature

Figure 21. Wing-body configuration obtained after fuselage tailoring.

\section{B. Fuselage tailoring of a lifting wing-body configuration}

When the configuration is producing lift at a given angle of attack $\alpha$ different from zero, the effective length $l_{e}$ of the $A_{e}$ distribution is different from the aircraft length $L$. The relationship between the flight axis $x$ and the horizontal one $x_{e}$ is defined as:

$$
x_{e}=x \cos (\alpha)-x \frac{\sin (\alpha)}{\tan (\mu)}
$$

Furthermore, to take into account the impact of lift on the design of an aircraft all the terms of Eq. (20) have to be considered. In the present study we use CFD to provide the lift contribution in the 


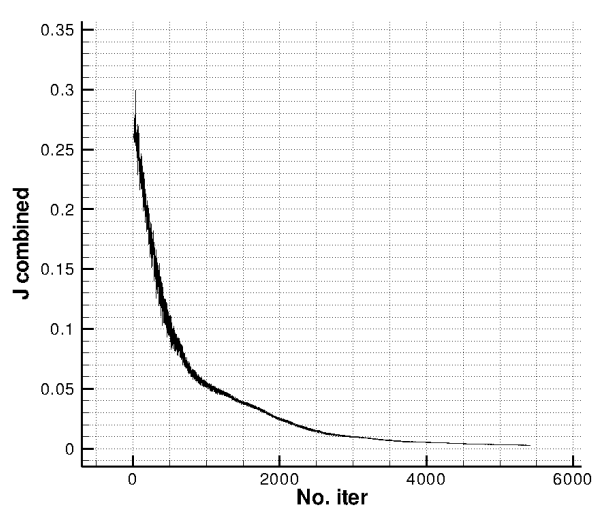

a) Cost function convergence history.

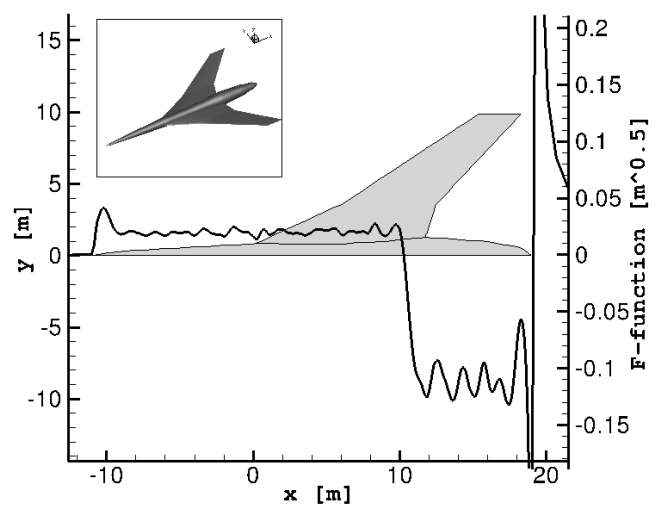

b) Shaped configuration. Geometry and corresponding F-function.

Figure 22. Shaped non lifting configuration.

form of skin pressure distribution. However, to avoid the call to a CFD code during the fuselage tailoring, two assumptions are made:

- the lift contribution originates from the wing component only;

- the wing lift distribution does not change significantly during fuselage shape modification.

The assumptions has been verified by a CFD study that has revealed that the term $A_{l}^{\text {fuse }}$ contributes to lift by less then $2 \%$ of the total $A_{l}$ distribution. Furthermore as long as the angle of attack is frozen, and with the fuselage radius modifications foreseen, the wing lift distribution variation should not be significant. The second approximation is made as the exposed wing surface may change during the fuselage tailoring but is assumed to be of limited extent. The two hypothesis are well satisfied if the initial configuration geometrical deviation from the target one is small enough. Using a reference wing-body geometry all the terms of the equation (20) are evaluated using CFD and geometry analysis. A new geometry can be defined using the approximation:

$$
A_{g}^{\text {fuse }}(x) \simeq A_{v}^{\text {fuse }}\left(x_{e}\right)=A_{e}^{\text {target }}\left(x_{e}\right)-A_{v}^{\text {wing }}\left(x_{e}\right)-A_{l}^{\text {wing }}\left(x_{e}\right)-A_{l}^{\text {fuse }}\left(x_{e}\right)
$$

The computational volumic mesh associated to the tailored geometry is then completely regenerated using an in-house analytical mesh tool. After an iteration of the fuselage tailoring 
process, all the equivalent area terms are re-evaluated with a CFD computation on the new configuration, and are used as starting point for the following fuselage tailoring iteration. The $A_{l}$ distribution, that has been evaluated only for two configurations, is then frozen during the tailoring iterations.

At this stage, a new target configuration that produce a flat-top ground signature has been designed using AIDA with the input values summarized in Table 9. The design flight conditions are: $M_{\infty}=1.6, \alpha=2^{\circ}, h=18,000 \mathrm{~m}$. The resulting unknown coefficients obtained for the optimal F-function modelling are summarized in Table 10.

\begin{tabular}{|cc||cc|}
\hline \hline Var. & Value & Var. & Value \\
\hline$W$ & $16,000 \mathrm{~kg}$ & $t$ & $0 \mathrm{~m}$ \\
$N, P$ & 1 & $\eta_{2}$ & 0 \\
$B_{1}, B_{2}$ & $0 m^{0.5} 0 \mathrm{ft}^{0.5}$ & $\eta_{3}$ & $\eta$ \\
$\eta$ & 0.5 & $y_{f}$ & $1.524 \mathrm{~m}$ \\
$\beta_{0}, \beta_{2}$ & 0 & $l_{e}$ & $28.67 \mathrm{~m}$ \\
\hline \hline
\end{tabular}

Table 9. AIDA input coefficients.

\begin{tabular}{|cc|}
\hline \hline Var. & Value \\
\hline$H$ & $0.00945 m^{0.5}$ \\
$C$ & $0.00362 m^{0.5}$ \\
$D$ & $0.00591 m^{0.5}$ \\
$y_{r}$ & $33.419 m$ \\
$\lambda$ & $21.362 m$ \\
\hline \hline
\end{tabular}

Table 10. Coefficients evaluated with AIDA.

The fuselage tailoring process adopts the same design variables as the the previous test case (41 radius sections) and the same initial geometry shown in Fig. 19c. The Nealder Mead simplex algorithm is adopted for the reduction of $J_{\text {comb }}$. The final configuration is obtained after a reduction of $J_{\text {comb }}$ by more than $80 \%$ in less than 2,000 iterations (see Fig. $24 \mathrm{a}$ and Fig. 24b).

The F-function shows an accurate agreement, in particular at the discontinuities. Several oscillations occur in the horizontal zero slope region of the function which are due to variations of curvature in $A_{e}$ (Fig. 23b), yet this accurately describes the target distribution. The main consequence of these oscillations is a near flat-top ground signature (Fig. 23c) with several small amplitude shocks. However, the tailored geometry (Fig. 24b) is not wavy but shows a significant reduction of the cabin volume. To control the cabin volume, an additional constraint could be taken into account in the optimization problem definition. 


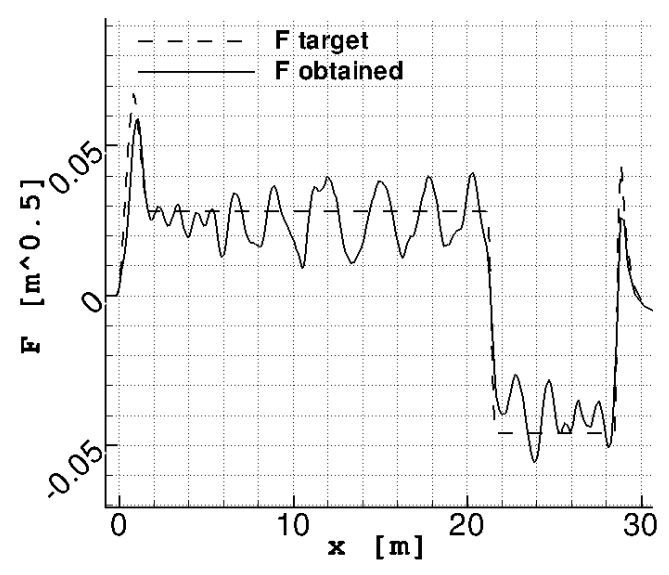

a) F-function.

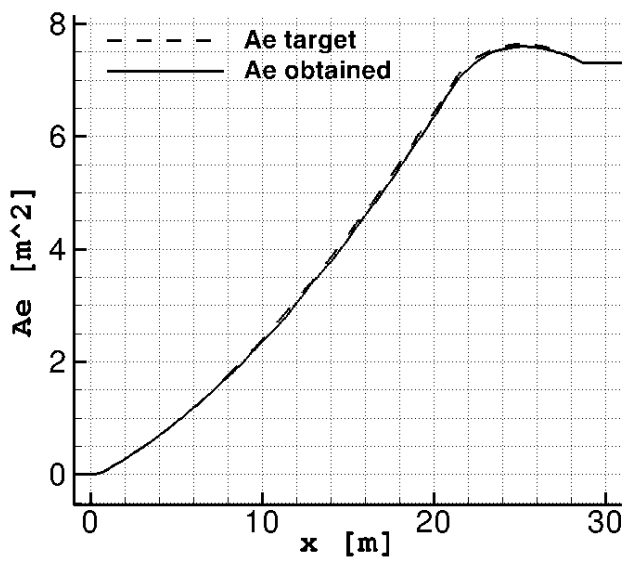

b) Equivalent Area.

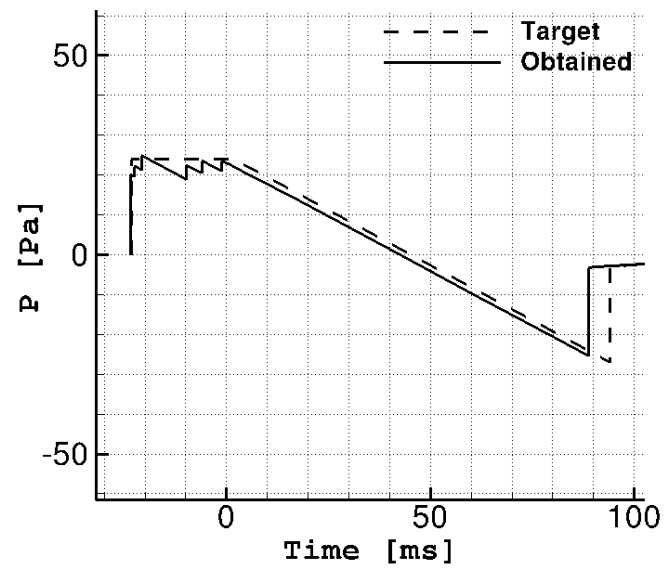

c) Ground Signature.

Figure 23. Wing-body lifting configuration obtained after fuselage tailoring.

\section{Conclusions}

The inverse design approach based on the shaped sonic boom theory has been investigated and was presented in this article. Although the direct shape optimization methods are widely used, the approach presented in this article is useful for preliminary design of low-boom configuration.

A module called AIDA (Acoustic Inverse Design Approach) has been developed to overcome the drawbacks of the classical shaped sonic boom minimization. An optimization-based procedure has been adopted to evaluate the coefficients of the F-function. The approach has the property, in contrast with other methods of the literature, to be applicable to different F-function parame- 


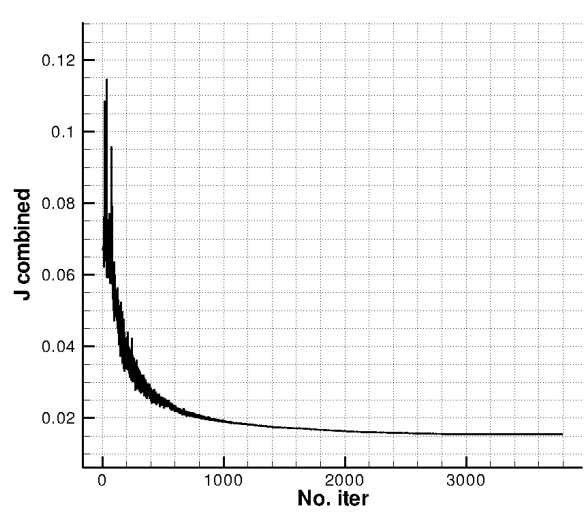

a) Cost function convergence history.

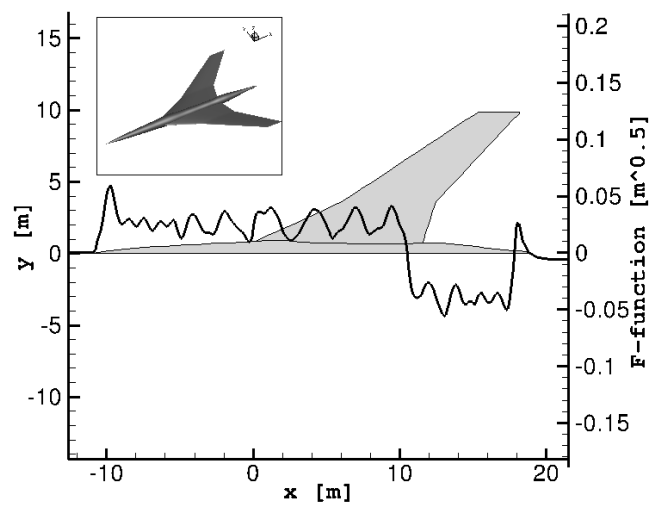

b) Shaped configuration. Geometry and corresponding F-function.

Figure 24. Shaped lifting configuration.

terizations without analytical reformulation of the problem. It enables also the introduction of a constraint on the front shock overpressure if the aircraft length constraint is relaxed.

A fuselage tailoring based on shape optimization is adopted with success on different test cases of increasing complexity to evaluate the geometrical area distribution that corresponds to a target equivalent area. Results show that the geometry parameterization has to describe in detail the regions that corresponds to the $F^{\prime}$ discontinuities. Furthermore a suitably chosen combination of the cost functions, based on the F-function and the equivalent area, provides an accurate approximation of the target ground signature with the final obtained tailored configuration.

The definition of a low-PLdB target function using an optimization process shows that it is possible to introduce with limited efforts relevant up-to-date acoustic metrics in the inverse design. Current methods in literature can define only the shape of the ground signature, while the introduction of relevant acoustic metrics can improve the inverse design methodology. The approach proposed based on optimization and an enriched F-function parameterization may also improve the shaped sonic boom theory introducing new metrics or trade-off with other disciplines such as aerodynamic performance.

Off-design conditions can impact negatively on the result provided by the inverse approach that defines a low-boom configuration for a set of conditions (Mach, altitude,...). An extension of the present work would be to focus on the sensitivity of the functions of interest with 
respect to the design variables aiming at performing robust design at a single or multiple flight conditions.

\section{References}

[1] Chambers, J.R., "Innovation in Flight: Research of the NASA Langley Research Center on Revolutionary Advanced Concepts for Aeronautics”, NASA SP-2005-4539, 2005.

[2] Seebass, R., and Woodhull, J.R., "History and Economics of, and Prospect for, Commercial Supersonic Transport," RTO AVT course on Fluid dynamics research on supersonic aircraft, 1998.

[3] Morgenstern, J., Norstrud, N., Stelmack, M. and Jha, P., "Advanced Concept Studies for Supersonic Commercial Transport Entering Service in 2030-35 (N+3)," AIAA paper 2010$5114,2010$.

[4] NASA LRC, "High-Speed Research: Sonic Boom, vol. I," NASA CP-3172, vol. 1, 1992.

[5] NASA LRC, "High-Speed Research: Sonic Boom, vol. II,” NASA CP-3173, vol. 2, 1992.

[6] Wlezien, R., Veitch, L., “DARPA Quiet Supersonic Platform Program,” AIAA paper 20020143, 2002.

[7] Ohnuki, T., Hirako, K., and Sakata, K., "National Experimental Supersonic Transport Project," in proceedings of the 25th ICAS, 2006.

[8] Welge, H.R., Nelson, C. and Bonet, J., "Supersonic Vehicle Systems for the 2020 to 2035 Timeframe," AIAA paper 2010-4930, 2010.

[9] Plotkin, K.J., "State of the Art of Sonic Boom Modeling," The Jo. of the Acoustical Society of America, Vol. III, No. 1, pp. 530-536, 2002.

[10] Alonso, J.J., and Colonno, M.R., "Multidisciplinary Optimization with Applications to Sonic-Boom Minimization”, Annual Review of Fluid Mechanics, Vol. 44, pp. 505-526, 2012. 
[11] Whitham, G.B., "The Flow Pattern of a Supersonic Projectile," Communications on pure and applied mathematics, Vol. 5, No.3, pp. 301-348, 1952.

[12] Walkden, F., "The Shock Pattern of a Wing-Body Combination, far from the Flight Path", Aeronautical quarterly, Vol. IX, pp. 164-194, 1958.

[13] Hunton, L.W., Hicks, R.M., and Mendoza, J.P., "Some Effects of the Mach Number and Geometry on Sonic Boom", NASA TN D-4214, 1967.

[14] Darden, C.M., "Limitations of Linear Theory for Sonic Boom Calculations", Journal of Aircraft, Vol. 30, No. 3, pp. 309-314, 1993.

[15] Cheung, S.H., Edwards, T.A., and Lawrence, S.L., "Application of CFD to Sonic Boom Near and Mid Flow-Field Prediction," NASA TM-102867, 1990.

[16] Kandil, O.A., and Ozcer, I.A., "Sonic Boom Computations for Double-Cone Configuration using CFL3D, FUN3D and Full-Potential Codes, "AIAA paper 2006-0414, 2006.

[17] Alauzet, F. and Loseille, A., "High-order Sonic Boom Modeling Based on Adaptive Methods," Journal of Computational Physics, Vol. 229, pp. 561-593, 2009.

[18] Wintzer, M., Nemec, M., and Aftosmis, M.J., "Adjoint-based adaptive mesh refinement for sonic-boom prediction," AIAA paper 2008-6593, 2008.

[19] Aftosmis, M.J., Nemec, M., and Cliff, S.E., "Adjoint-Based Low-Boom Design with Cart3D," AIAA paper 2011-3500, 2011.

[20] Loseille, A., and Lohner, R., "Anisotropic Mesh Generation Application To High-Fidelity Simulation in CFD," AIAA paper 2010-0169, 2010.

[21] Plotkin, K., and Page, J., "Extrapolation of Sonic Boom Signatures from CFD Solution," AIAA paper 2002-0922, 2002.

[22] Rallabhandi, S. K., and Dimitri, N. M., "Sonic boom modelling under atmospheric uncertainty," AIAA paper 2004-4650, 2004. 
[23] Yamaguchi, H., and Nakamura, Y., "Optimization of Low Boom Configuration of SST by Genetic Algorithm," AIAA paper 98-2899, 1998.

[24] Alonso, J.J., Kroo, I.M., and Jameson, A., "Advanced Algorithms for Design and Optimization of Quiet Supersonic Platforms," AIAA paper 2002-0144, 2002.

[25] Farhat, C., Maute, K., Argrow, B., and Nikbay, M., "Shape Optimization Methodology for Reducing the Sonic Boom Initial Pressure Rise”, AIAA Journal, Vol.45, No. 5, pp. 1007-1018, 2007.

[26] Seebass, R., and George, A.R., "Sonic-Boom Minimisation", Journal of the acoustical society of America, Vol 51, No.2, 1972, pp 686-694.

[27] Barger, R.L. and Adams, M.S., "Fuselage Design for a Specified Mach-Sliced Area Distribution”, NASA TP-2975, 1990.

[28] Mack, R. J., and Needleman, K. E., “A Semi-Empirical Method for Obtaining Fuselage Normal Areas from Fuselage Mach Sliced Areas”, NASA TM-4228, 1991.

[29] Makino, Y., Aoyama, T., Iwamiya, T., Watanuki, T., and Kubota, H., "Numerical Optimization of Fuselage Geometry to Modify Sonic-Boom Signature”, Journal of Aircraft Vol. 36, No. 4, pp. 668-674, 1999.

[30] Li, W., Shields, W. and Le, D., "Interactive Inverse Design Optimization of Fuselage Shape for Low-Boom Supersonic Concepts", AIAA paper 2008-136, 2008.

[31] Palacios, F., Alonso, J.J., Colonno, M., Hicken, J., Lukaczy, T., “Adjoint-based Method for Supersonic Aircraft Design using Equivalent Area Distributions," AIAA Paper 2012-269, 2012.

[32] Pawlowski, J. W., Graham, D. H., Boccadoro, C. H., Coen, P. G., and Maglieri, D. J., “Origins and Overview of the Shaped Sonic Boom Demonstration Program," AIAA Paper 2005-5, 2005. 
[33] Honda, M., and Yoshida, K., "D-SEND Project for Low Sonic Boom Design Technology," Proceedings of 28th ICAS, 2012.

[34] Petty, J.S., "Lower Bounds for Sonic Boom Considering the Negative Overpressure Region," Journal of Aircraft, Vol. 7, No. 4, pp. 375-377, 1970.

[35] Hayes, W.D., Gardner, J.H., Coughey, D.A., and Weiskopf, F.B., “Theoretical Problems Related to Sonic Boom," NASA SP-255, 1970.

[36] Howell, C.S., Sigalla, A., and Kane, E.J., "Sonic Boom Considerations in Aircraft Design. Aircraft Engine Noise and Sonic Boom,” AGARD CP No. 42, pp. 301-307, 1969.

[37] George, A.R., "Lower Bounds for Sonic Booms in the Midfield," AIAA Journal, Vol. 7, No. 8, pp. 1542-1545, 1969.

[38] Jones, L.B., "Lower Bounds for the Pressure Jumps of the Shock Waves of a Supersonic Transport of Given Length,” Aeronaut. Quart., Vol. XXIII, pt. 1, pp. 62-76, 1972.

[39] Darden, C.M., "Sonic-Boom Minimisation with Nose-Bluntness Relaxation," NASA TP1348, 1979.

[40] Rallabhandi, S.K., and Mavris, D.N., "Sonic Boom Minimization Using Inverse Design and Probabilistic Acoustic Propagation,” Journal of Aircraft, Vol. 43, No. 6, pp. 1815-1828, 2006.

[41] Mack, R.J., “A Supersonic Business-Jet Concept Designed for Low Sonic Boom,” NASA TM-212435, 2003.

[42] Haas, A., and Kroo, I., "A Multi-Shock Inverse Design Methid for Low-Boom Supersonic Aircraft," AIAA paper 2010-843, 2010.

[43] Plotkin, K.J., Rallabhandi, S.K., Li, W., "Generalized Formulation and Extension of Sonic Boom Minimization Theory for Front and Aft Shaping," AIAA paper 2009-1052, 2009.

[44] Higgins, T.H., "Psychophisical Tests of Potential Design/Certification Criteria for Advanced Supersonic Aircraft," NTIS AD-A009 296, 1975. 
[45] Makino, Y., and Kroo, I., "Robust Objective Functions for Sonic-Boom Minimization,” Journal of aircraft Vol. 43, no.5, pp. 1301-1306, 2006.

[46] Sullivan, B.M., "Human Response to Simulated Low-Intensity Sonic Booms ,"proceeding of NOISE-CON 2004, Baltimore, Maryland, 2004.

[47] Stevens, S., "Perceived Level of Noise by Mark VII and Decibels(E)," J. Acoust. Soc. Am. Vol. 54, No. 2, pt.2, pp 575-601, 1972.

[48] Taylor, A.D., “The TRAPS Sonic Boom Program ,'NOAA Technical memorandum ERL-ARL$87,1980$.

[49] Hayes, W.D., Haefeli, R.C., and Kulsrud, H.E., "Sonic Boom Propagation in a Stratified Atmosphere with Computer Program,” NASA CR-1299, 1969.

[50] George, A., and Plotkin, K.J., "Sonic Boom Waveforms and Amplitudes in a Real Atmosphere," AIAA Journal, Vol. 7, No.10, pp. 1978-1981, 1969.

[51] Cambier, L., and Gazaix M., "An Efficient Object-Oriented Solution to CFD Complexity," AIAA paper 02-0108, 2002.

[52] Coulouvrat, F., "The Challenges of Defining an Acceptable Sonic Boom Overland," AIAA paper 2009-3384, 2009.

[53] Rasheed, K., “GADO: A Genetic Algorithm for Continuous Design Optimization ”, Department of Computer Science, Rutgers, The State University of New Jersey, Technical Report DCS-TR-352, 1998.

[54] Sandia National Laboratoires. "DAKOTA, A Multi-level Parallel Object-Oriented Framework for Design Optimization, Parameter Estimation, Uncertainty, Quantification, and Sensitivity Analysis", Reference manual, 2007.

[55] Kang, J., and Pierce, A.D., "The Effect of Molecular Relaxation Processes in Air on the Rise Time of Sonic Booms", Proceedings International Congress on Recent Developments in Airand Structure-Borne Sound and Vibration, Auburn, AL, Mar. 6-8, Vol. 1, pp. 97-104, 1990. 
[56] Elmer, K.R., and Joshi, M.C., "Variability of Measured Sonic Boom Signatures: Volume 1", NASA CR-191483, 1994. 